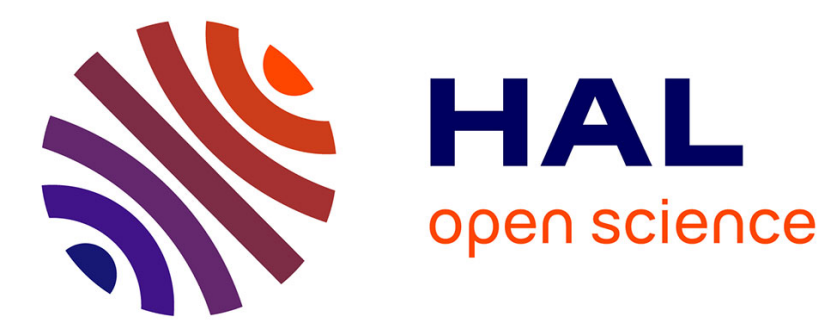

\title{
Processes involving few degrees of freedom in the frame of Intranuclear Cascade approaches
}

\author{
J Cugnon, A Boudard, J.C. David, S Leray, D Mancusi
}

\section{To cite this version:}

J Cugnon, A Boudard, J.C. David, S Leray, D Mancusi. Processes involving few degrees of freedom in the frame of Intranuclear Cascade approaches. The European Physical Journal Plus, 2016, 131 (5), pp.169 - 169. 10.1140/epjp/i2016-16169-4 . hal-01467675

\section{HAL Id: hal-01467675 \\ https://hal.science/hal-01467675}

Submitted on 16 Feb 2017

HAL is a multi-disciplinary open access archive for the deposit and dissemination of scientific research documents, whether they are published or not. The documents may come from teaching and research institutions in France or abroad, or from public or private research centers.
L'archive ouverte pluridisciplinaire HAL, est destinée au dépôt et à la diffusion de documents scientifiques de niveau recherche, publiés ou non, émanant des établissements d'enseignement et de recherche français ou étrangers, des laboratoires publics ou privés. 


\title{
Processes involving few degrees of freedom in the frame of Intranuclear Cascade approaches ${ }^{\star}$
}

\author{
J. Cugnon ${ }^{1, a}$, A. Boudard ${ }^{2}$, J.-C. David ${ }^{2}$, S. Leray ${ }^{2}$, and D. Mancusi ${ }^{2}$ \\ 1 AGO Department, University of Liège, allée du 6 Août 19, bât. B5, B-4000 Liège 1, Belgium \\ ${ }^{2}$ CEA/Saclay, Irfu/SPhN, F-91191 Gif-sur-Yvette, France
}

Received: 21 December 2015 / Revised: 26 February 2016

Published online: 26 May 2016 - (C) Società Italiana di Fisica / Springer-Verlag 2016

\begin{abstract}
This article focuses on spallation reactions, i.e. interactions of energetic nucleons, basically with a kinetic energy in the $100 \mathrm{MeV}$ to a few $\mathrm{GeV}$ range, with a target nucleus. These processes are described rather successfully by the so-called Intranuclear Cascade (INC) plus evaporation models. They can be viewed as a first stage of nucleon-nucleon collisions, ejecting fast particles, followed by evaporation of slow particles from the target remnant. These cascade + evaporation models have, now, globally reached a high level of predictive power, owing in particular to successive research programs. The present work, which is an outcome of one of these programs, the recent European Union ANDES research program, deals with a set of reactions (or observable quantities), which can be due to a single collision, such as the one-nucleon removal reactions or the quasi-elastic elastic process. A survey of the experimental data is presented, which allows to clearly point out that, often, the INC models are unsatisfactory for the description of these peculiar events, whereas they are rather successful for the rest of the experimental data. This paradoxical situation is tentatively related to quasi-particle effects which are neglected in INC models.
\end{abstract}

\section{Introduction}

In the past ten years, there has been a revival in the study of spallation reactions, largely triggered by efforts devoted to the development of various applications: transmutation of nuclear waste $[1,2]$, new spallation neutron sources for condensed matter and material studies [3], simulation of experimental set-ups in nuclear and particle physics [4], production of rare isotopes [5], protection against radiation near accelerators and in space missions [6], interaction of cosmic rays in the atmosphere [7], cancer hadrontherapy [8], etc. These applications involve interactions of energetic particles with macroscopic bodies (the macroscopic spallation processes), which in turn result from the interaction of nucleons or light clusters with nuclei (the microscopic spallation reactions, simply referred here as spallation reactions). These reactions are most often described by two-stage models. In the first stage, the incoming particle gives rise to a sequence of hard collisions which eject a few fast particles, mainly nucleons, but also light charged particles (lcp), to a lesser extent. At the end of this stage, the target remnant is left with a residual excitation energy, that it further releases on a much slower pace, very much akin to evaporation. Quite often, this stage is described by statistical evaporation models, whereas the first stage is described with the help of intranuclear cascade (INC) models. Basically, INC models are quasi-classical models that simulate this first stage by a succession of independent binary collisions resulting from close encounters of nucleons, which otherwise are propagating freely along straight lines. These models work rather well when the conditions for the independence of successive collisions are met, basically when the incident energy is larger than, say $150 \mathrm{MeV}$, and smaller than a few $\mathrm{GeV}^{1}$. Actually, recent studies [9] have shown that cascade models seem to work well at much lower incident energy, i.e. down to a few tens of MeV. This phenomenon is not well understood. See ref. [10] for an interesting discussion of this point.

\footnotetext{
* Contribution to the Focus Point on "Nuclear data for energy" edited by S. Leray.

a e-mail: cugnon@plasma.theo.phys.ulg.ac.be

1 This upper limit may be understood as roughly corresponding to the onset of sub-nucleonic degrees of freedom. This limit should however be considered as a rather conservative one. It is indeed probable that the independence of collisions is still prevailing at higher energy, even if collisions then involve hadronic resonances.
} 
In any case, within the accepted limit of validity, cascade models are working quite well, globally. This is testified by the intercomparison of cascade + de-excitation models which has been carried out a few years ago by the IAEA [9]. Predictions of the models have been confronted with measurements of many different observables, including double differential nucleon, pion and light charged particle production cross sections, particle multiplicities and residue production cross sections, for various targets and for incident kinetic energy ranging from $150 \mathrm{MeV}$ to $3 \mathrm{GeV}$. Analyses and evaluations of this intercomparison can be found in refs. [11,12]. The rather high predictive power of present cascade + de-excitation models results, in particular, from a coordinated effort in the last fifteen years which took place in successive European Union research programs, principally HINDAS [13], NUDATRA [14] and ANDES [15]. However, when the performances were analyzed in detail in the frame of the recently completed ANDES program, a somewhat surprising phenomenon has progressively been put in evidence.

Even when the conditions of independent collisions are met, cascade models are relying on simplifying tools semiclassical tools. Basically, binary collisions are viewed as instantaneous phenomena occuring when the relative distance of approach is sufficiently small in comparison with the square root of the nucleon-nucleon cross section divided by $\pi$. In particular, this approach neglects quantum motion and possible interferences. Furthermore, nucleons are always considered as on-shell entities, which is certainly an approximation. Since INC models offer simplified pictures of binary collisions, they might naively be expected to work worse and worse for observables corresponding to more and more collisions. Contrarily to this expectation, INC models seem to work better for many-collision events than for single-scattering events. This was noticed regularly, but little attention was paid to this observation $[16,18,19,12$, 20-23,10,24]. For instance, in proton-induced reactions, the spectrum of neutrons emitted at zero degree exhibits a high energy peak (the so-called quasi-elastic peak), which corresponds to neutrons that are emitted forward in the first collision made by the incident proton. The low-energy part of this spectrum (roughly in the $20-100 \mathrm{MeV}$ interval) is due to the emission of neutrons in events where the incident proton has undergone several collisions [16]. It is now a rather well established fact that INC models have a tendency to better describe this part of the spectrum than the high-energy (single-scattering) part. This could be explained, as also alluded to later, by the possible cancellation of interference terms in many-collision events. This point, although still conjectural, had been advanced by the pioneers of the INC model [17]. Nevertheless, the difficulty of INC models to cope with single-scattering observables has also been put to light by recent works on the so-called one-proton removal cross sections, or, in other words, cross sections which are related to exclusive $(\mathrm{p}, 2 \mathrm{p})$ reactions. In these reactions, the incoming proton makes a single collision with a proton of the target, the two protons escape from the nucleus, leaving a remnant with an excitation energy which is too small for allowing subsequent particle evaporation (see below for more detail). It has been recognized that the cross sections predicted by INC models are systematically too large, by a factor of 2 or 3 [25,26]. This observation is rather puzzling, since the bulk of the residue production cross sections, involving a few or many collisions, are generally reproduced rather well, with a much better accuracy. See ref. [9] for detail.

This surprising result, as well as similar results related to events with very few collisions (one or two at the most) have received attention during the activity of some of the authors of this paper within the framework of the ANDES EU research program [15]. A global view of the performances of INC models with respect to reactions involving very few degrees of freedom has progressively emerged from this research program. It is the purpose of this note to elaborate on this activity and to make a review of the way some selected observables are connected with single scattering mechanisms, how these mechanisms are described by INC models and how the INC model compare with quantum multiple scattering approaches when such a comparison is possible. On several aspects, we will also try to propose some improvements of INC models. For practical purposes, we will concentrate on the Liège Intranuclear Cascade model [24] (INCL), because we have participated to the continuous improvement of this model during the last fifteen years. Most of the time, this model will be used in connection with the so-called ABLA07 evaporation-fission model [27]. However, we think that what we are going to present affects rather generic aspects of current INC models and is largely independent of the evaporation models, for reasons that will be explained later. Occasionaly, results that are more specific to INCL model will be presented. This specificity will be acknowledged.

The paper is divided as follows. Section 2 contains a quick presentation of the INCL model. We will not describe here in detail the associated ABLA07 model, since, as explained later, the evaporation model plays little role in the kind of processes under investigation in this paper. In sect. 3, we will present a classification of events involving only a few collisions or a few degrees of freedom. The connection with reaction mechanisms based on a definite and small number of collisions will be established. This aims at preparing the comparison with INC predictions and at possibly identifying dynamical features which are omitted in INC models. This will cover inclusive quasi-elastic and quasi-inelastic scatterings, coherent excitation to target low-lying states and production of residues that are not very different from the target. Section 4 presents phenomenological simple relations between some residue production cross sections, which enlight the connection between these cross sections and the underlying mechanisms in term of collisions. These connections are not transparent from INC results. In sect. 5, we compare INC models with quantum single scattering theories in the case of quasi-elastic scattering. Discrepancies will be analyzed in terms of features that are neglected in INC models. In sect. 6, we will quickly review another ouput of the ANDES Collaboration, the effect of single-scattering events on some special observables in macroscopic applications. Section 7 contains our conclusion. 


\section{Relevant features of INC models}

For the sake of clarity, we briefly review here the features of INC models. We restrict here to aspects that are relevant for the purpose of this paper. We will successively review generic features of the INC models and features which are specific of INCL. We restrict to nucleon-induced reactions, for simplicity.

\subsection{Generic features}

The generic features of INC models are:

1) Preparation of the event. The target is prepared with proper spatial and momentum density distributions. There are generally two ways to realize that preparation, either by setting A nucleons at random in a way consistent with the spatial and momentum nucleon densities, or simply considering the target nucleus as a continuum in space gifted with an underlying Fermi sea. Particles are supposed to feel an attractive mean potential well. The two methods may give different results for some observables (not really those which are of interest in this paper), but both are using realistic phenomenological density distributions. The incident particle is launched to the target with the appropriate velocity and with a random impact parameter.

2) Propagation of particles and collisions. In the first kind of models, all particles are set into motion and followed in time. If two particles are coming close enough, i.e. if their minimum relative distance of approach $d_{\text {min }}$ satisfies $\pi d_{\text {min }}^{2} \leq \sigma_{\text {tot }}$, where $\sigma_{\text {tot }}$ is the total particle-particle cross section, they are allowed to collide, provided the final states are not blocked by the Pauli principle. In the second kind of models, the incident particle is given a free path, after which it collides with a nucleon promoted from the underlying Fermi sea. Collisions are also possibly Pauli-blocked. Since the mean free path is also determined by $\sigma_{\text {tot }}$, collisions are more or less realized in a similar way in the two types of model. Elastic and inelastic collisions are considered. Propagation is then resumed untill the next collision. In the second type of models, promoted nucleons are also propagated.

3) Other avatars. Besides collisions, free propagation is interrupted by other avatars ${ }^{2}$. Most often, two other types of avatars are introduced: decay of unstable particles ( $\Delta$-resonances) and reflection/transmission on the nuclear surface.

4) Stopping of the events. Usually, the simulation of an event is stopped when a criterion, generally based on the energy of the nucleons inside the target, is fulfilled. After the stopping, the excitation energy of the remnant is evaluated, generally on basis of the energy of particle-hole excitations caused by the collision process. All the properties of the remnant can be passed to the evaporation code.

\subsection{Specific features of INCL4}

The INCL model has been in continuous evolution during the last ten years. The basic formulation, dubbed INCL4.2, has been published in ref. [19]. The next landmarks have been released in ref. [24] for INCL4.6 and in ref. [28] for $\mathrm{INCL}++$.

Let us first discuss the specific features which are common to all these versions. INCL deals with nucleons which are on-shell: there is a one-to-one correspondance between the energy and the momentum of the nucleons, even if they experience a potential, which is always supposed to be a time-like quantity. Also, INCL is a time-like model where all particles are followed simultaneously in space-time. The Pauli blocking of collisions is operating in phase space, with Pauli blocking factors involving the phase space density around the nucleons in the final state of the two-body collisions. A unique feature of INCL is the stopping criterion. Whereas most models stop when the total energy or the energy of the most energetic particle in the target comes below a chosen value, the time at which INCL is stopped is determined self-consistenly, by looking at the degree of randomisation of the target. Details can be found in ref. [19]. In addition, the coupling to evaporation is not subject to the introduction of a preceding pre-equilibrium module. Another specific feature is the fact that INCL accomodates light composite nuclei (up to ${ }^{4} \mathrm{He}$ particles) as incident particle. However, in INCL4.2, the implementation of this feature is rather crude.

The INCL4.6 version improves on the INCL4.2 version on several points. For the sake of our discussion here, we single out three of them (for more detail, see ref. [24]): i) it introduces the production of light charged clusters through the implementation of a dynamical surface coalescence model, ii) the binding of the nucleons inside an incident cluster is taken into account, iii) the treatment of soft collisions has been improved.

Finally, the version INCL ++ , besides providing with a $\mathrm{C}++$ version of the numerical code, improves considerably on the treatment of the dynamics of collisions induced by light clusters (now allowing incident masses up to 18).

\footnotetext{
${ }^{2}$ We use the term "avatar" for these changes of motion in the realization of a single incident particle-target interaction process and devote the word "event" for realizations of such complete particle-nucleus interactions. Grossly speaking, an event is made of several avatars.
} 
We recall that, in all the three versions of INCL mentioned above, the inelastic nucleon-nucleon collisions are assumed to be due to the excitation of the Delta resonance. Actually the following reactions are considered: $N N \rightleftharpoons N \Delta$, $\Delta \rightleftharpoons \pi N$. This description of the inelastic collisions seems to be satisfactory up to at least 2 GeV incident energy [19].

\subsection{Foundations of INC models}

INC models have developed on a rather heuristic way, progressively including the main features of the nuclear dynamics (nucleons moving freely between well separated collisions, in a static and constant potential well containing a nucleon Fermi gas), owing to simple simulation tools and without reference to a definite theoretical framework. Nevertheless, there is some theoretical foundation of INC models, which relies on the so-called nuclear transport theory. The latter is basically a way to reformulate the full quantum many-body problem in a form which is amenable to approximations suitable to the description of the gross features of nuclear dynamics. The derivation of the nuclear transport equations may be done in several ways. Either starting from the Green functions formalism, or from the von Neumann equation [29-31], these theories arrive more or less at the same basic evolution equation, namely

$$
\begin{aligned}
\left(\frac{\partial}{\partial t}+\frac{\boldsymbol{p}}{m} \cdot \boldsymbol{\nabla}-\boldsymbol{\nabla} U \cdot \boldsymbol{\nabla}_{p}\right) f(\boldsymbol{r}, \boldsymbol{p}, t)= & \pi \hbar^{2} \int \frac{\mathrm{d}^{3} p_{2}}{(2 \pi)^{3}} \int \frac{\mathrm{d}^{3} p_{3}}{(2 \pi)^{3}} \int \frac{\mathrm{d}^{3} p_{4}}{(2 \pi)^{3}}|G(12 \rightarrow 34)|^{2} \\
& \times\left\{f_{3} f_{4}(1-f)\left(1-f_{2}\right)-f f_{2}\left(1-f_{3}\right)\left(1-f_{4}\right)\right\} \delta^{3}(\boldsymbol{p}) \delta(e(p)),
\end{aligned}
$$

where $f_{i}=f\left(\boldsymbol{r}, \boldsymbol{p}_{i}, t\right)$. In this equation, $U$ is the nuclear mean field, determined by the distribution $f$ itself, $G$ is a medium-corrected transition matrix for the nucleon-nucleon collisions and the delta functions stand symbolically for momentum and energy conservation in collisions. In the simplest approximation, $U$ is given by

$$
U(\boldsymbol{r})=\int \mathrm{d}^{3} \boldsymbol{r}^{\prime} \int \mathrm{d}^{3} \boldsymbol{p}^{\prime}\left\langle\boldsymbol{r} \boldsymbol{r}^{\prime}|G| \boldsymbol{r} \boldsymbol{r}^{\prime}\right\rangle f\left(\boldsymbol{r}^{\prime}, \boldsymbol{p}^{\prime}, t\right) .
$$

The function $f(\boldsymbol{r}, \boldsymbol{p}, t)$ is the Wigner transform of the one-body density and, for our purpose, can be viewed as the distribution function of the nucleons in phase space. Equation (1) reduces the nuclear dynamics to the evolution of the one-body phase space density due to a drift term (left member) and to a collision term (right member). This transport equation is obtained after use of three main assumptions:

1) Closure approximation: the two-body correlations are unimportant in the collision term.

2) Low-gradient approximation: for this approximation to be valid, it is required, in particular, that the mean potential $U$ is a smooth function on the spatial extension of $f$, or more or less equivalently, on the spatial extension of the particle wave functions.

3) Independence of collisions: this means that, in an elementary collision, the scattering wave function becomes asymptotic before the next collision takes place.

To be complete, another approximation has been called for, which amounts to assuming that particles are on the mass shell (there is a one-to-one relation between $e$ and $p, e=p^{2} / 2 m+U$ in the case above). A more sophisticated version of eq. (1) can be obtained [29] which involves quasi-particles. Their energy is related to the momentum by a distribution of $e$ for a given $p$. This introduces an extra (energy) variable in $f$ and in the collisional integral.

Equation (1) having the classical form of a transport equation with a drift term and a collision term (rhs), it is tempting to solve this equation by simulations. This is an accurate method for the drift term. Moreover, it has been shown that the INC procedure amounts to evaluate the collisional integral by Monte Carlo method [32,33]. The whole procedure thus provides an accurate handling for averages over events. Note however that, in INCL, $U$ is a constant square well potential (with momentum-dependent depth and range). Between collisions, nucleons are moving along straight lines. This provides INCL with a considerable practical advantage in terms of computing time efficiency, since the propagation of nucleons between collisions is trivial [19].

Since we focus in this paper on the validity of the multi-scattering picture, the most crucial approximation for our discussion is evidently approximation 3 mentioned above ${ }^{3}$. There is some kind of agreement that this approximation is valid under the following conditions:

$$
\pi \lambda_{B} \ll r_{s} \lesssim d
$$

\footnotetext{
${ }^{3}$ In the INCL model, approximation 2 may also raise some concern difficulties, since this model uses average potential wells with a sharp surface [19], (although the average density is smooth), but it may be hoped that the use of stochastic reflection and transmission coefficients at the nuclear surface embodies, for the global particle currents at least, the effects of the propagation of the particles in a realistic surface.
} 
The first inequality ensures that the de Broglie wavelength for the relative motion in the entrance channel is smaller than the size of the collision zone around a nucleon, which justifies the use of classical trajectories. It is customary to consider the quantity $r_{s}$ as the scattering length. This is justified at low energy. In our case, it is more appropriate to take $r_{s}$ as the range of the nuclear forces. The second inequality, where $d$ is the mean distance between nucleons in a target nucleus, guarantees the independence of the successive collisions. In practice, the first inequality is satisfied whenever the incident energy is larger than $200 \mathrm{MeV}$. The second inequality is fulfilled in nucleon-induced spallation reactions, but only marginally (see for instance ref. [10]).

Let us close this Section by a short discussion of the classical nature of INC models. Often it is claimed that these models are purely classical, as are also essentially transport equations like eq. (1). As a matter of fact, there is no $\hbar$ in this equation. Of course, quantum effects are nevertheless present. Some of them are hidden in the transition matrix, but when the latter is expressed in terms of cross sections and if these cross sections are taken from phenomenology, the quantum nature of the transition matrix is no more visible or effective. Another quantum effect, the Pauli principle, is present in eq. (1) through the $(1-f)$ factors. In addition, INC models are taking care, by simulation of course, of other quantum effects. Final states of binary collisions are determined stochastically and particles are transmitted or reflected stochastically at the nuclear surface. On the other hand, quantum motion interferences and, as already mentioned, the quasi-particle nature of nucleons in interactions are neglected.

To summarize for the sake of the present discussion, possible failures of INC models may arise because the conditions of validity of independent collisions are not fulfilled or because these models lack to describe quantum interferences and quasi-particle effects.

\section{Classification and characterization of events involving few degrees of freedom}

\subsection{Inclusive nucleon production in the quasi-elastic and quasi-inelastic regimes}

The presence of a well-pronounced peak in inclusive proton cross sections at an energy close to the incident energy for proton-induced reactions, as shown in fig. 1, stands as a fingerprint of the production of the outgoing proton from a single collision between the incoming proton and a target proton (or neutron) with basically the same kinematics as a free proton-proton (or proton-neutron) collision. The same phenomenon is also observed in the more often investigated neutron production spectra in proton-induced reactions, as illustrated in fig. 2. This time, the peak in the neutron spectra results from a single collision between the incoming proton and a target neutron, which is kicked off in the incident direction. For the colliding pair, this corresponds however to a backward scattering in their c.m. frame (this can as well be viewed as a forward charge exchange scattering).

The position of the maximum of this so-called quasi-elastic peak does not exactly correspond to the value of the incident energy (it lies some $40 \mathrm{MeV}$ below in the examples of figs. 1 and 2). This comes mainly from the fact that the collision is not a totally free one, for at least three reasons: the target partner is bound, it has a Fermi motion inside the target nucleus and the latter has to recoil. There may be other reasons, as alluded below. Furthermore, the quasi-elastic peak has a width, which is expected to reflect the Fermi motion inside the target nucleus.

Although the arguments above indicate that nucleons of the quasi-elastic peak are originating from the first collision induced by the incident proton, this does not mean that they are produced in single scattering events. Indeed the quasi free kinematics implies that the proton ejected in the forward collision after the first collision has to avoid making further collision, but allows the partner nucleon to make other subsequent collisions. In other words, the cross section corresponding to the quasi-elastic peak is not simply equal to the cross section for making at least a collision (we will see how to define the latter, in the frame of collisional models). One has to account for the fact that the forward outgoing nucleon should avoid further collision. This cross section is not equal to the cross section for making a single collision either, since the unobserved partner nucleon may make several collisions. Note that there are two contributions to the quasi-elastic peak in proton spectrum: the first collision is either an elastic $p p$ collision or an elastic $p n$ collision, with the proton emitted at forward angles. The quasi-elastic peak in neutron spectra is solely due to elastic $p n$ collisions with the neutron emitted at forward angles.

The quasi-elastic peak in proton spectra seems to be rather well reproduced by INCL, as it is illustrated in fig. 1 and in ref. [19]. The magnitude, the shape and the angular dependence seems to be rather well described. This holds as long as the incident energy is larger than, say $200 \mathrm{MeV}$. For lower incident energy, the quasi-elastic peak is not really present in the spectra or is roughly dissolved in a background. Actually, it has been checked (by INC models) that, at high energy (larger than $\sim 500 \mathrm{MeV}$ ), the protons in the quasi-elastic peak are really coming from the first scattering made by the incident proton [16]. There is practically no other contribution. At smaller incident energy, the contributions to the spectra coming from particles issued from first, second, third..., collisions are overlapping. This is expected from the fact that the Fermi momentum is getting closer and closer to the size of the incident momentum. 


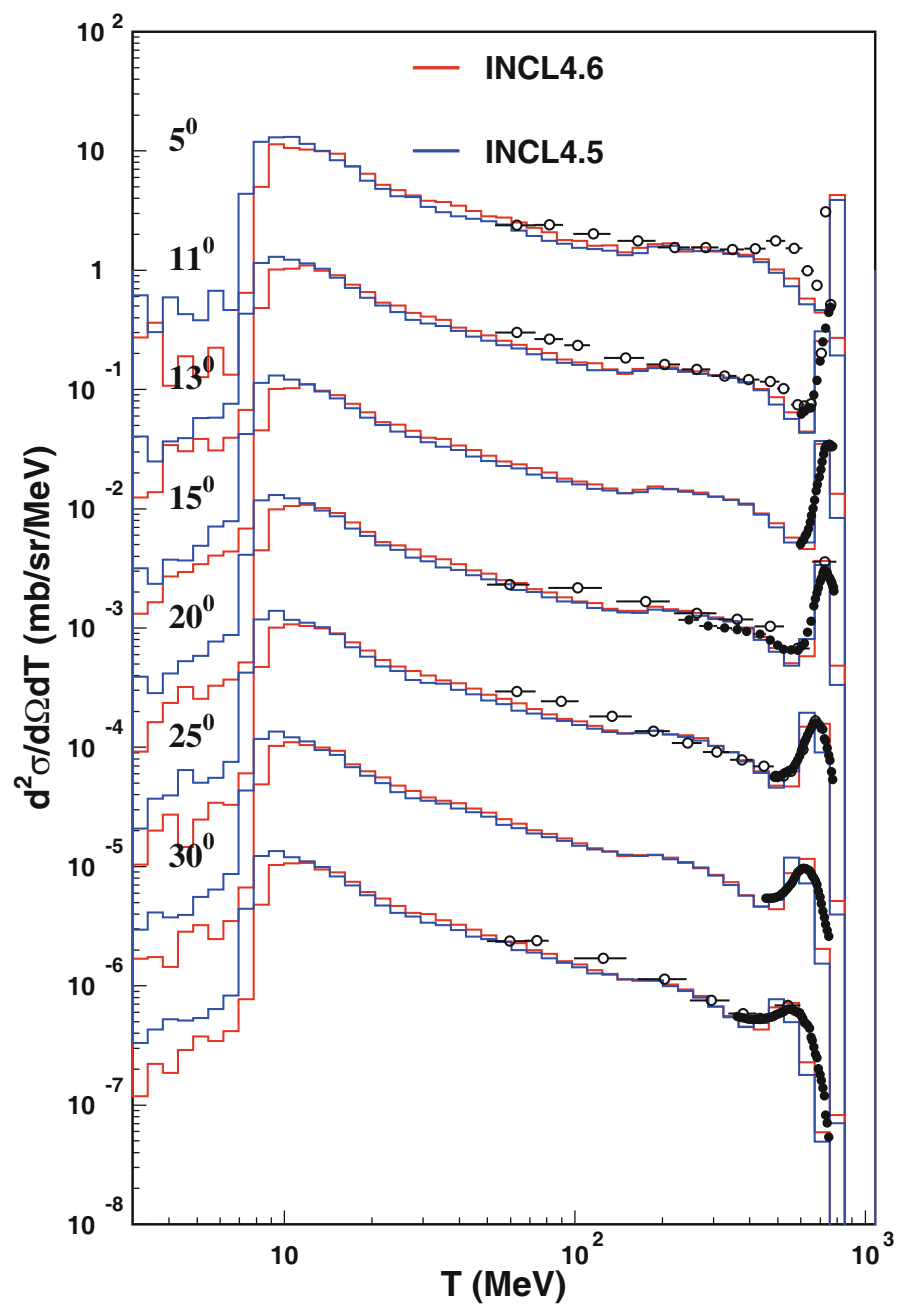

Fig. 1. Proton double differential cross sections for $p(800 \mathrm{MeV})+\mathrm{Pb}$ reactions, as functions of the kinetic energy of the emitted proton, at different angles, indicated on the figure. For the sake of clarity, the spectra at the various angles have been multiplied by $10^{0}, 10^{-1}, 10^{-2}$, etc., for successive angles in increasing order. Data (symbols) are from refs. [34,35]. They are compared with the predictions of INCL4.5 (blue histograms) and INCL4.6 (red histograms). The difference between the INCL4.6 version of INCL and its forerunner INCL4.5 [23] is of no importance in this paper. For more detail, see ref. [24].

As for the quasi-elastic peak in neutron spectra (for proton-induced reactions), the predictions of INCL are less good, even at large incident energy, as can be seen in ref. [9] and in fig. 3. The position of the peak is located at too high a neutron energy, the width is underestimated by a factor 2 or so and the total intensity is slightly underestimated by 0 to $20 \%$, depending upon the cases. Once again, this is true for high incident energy. For low incident energy, the predicted peak is dissolved in the background, which is more or less satisfactorily reproduced.

Let us say a few words about the quasi-inelastic peaks. They can be seen in the proton spectra and neutron spectra displayed in figs. 1, 2 and 3 at a proton or a neutron energy roughly $250 \mathrm{MeV}$ smaller than the incident kinetic energy. The quasi-inelastic peak occuring in proton spectra corresponds to events where the first collision is a $p p \rightarrow p \Delta^{+}$(or $p n \rightarrow p \Delta^{0}$ ) process with the emitted proton appearing at forward angles, with no restriction on what happens to the $\Delta^{+}$(or to the $\Delta^{0}$ ). There are possibly other contributions: $p p \rightarrow p \Delta^{+}\left(\right.$or $p p \rightarrow n \Delta^{++}$) with the $\Delta^{+}$(or $\Delta^{++}$) being emitted at forward angles and decaying with the emission of a proton in the forward direction. These contributions are however very small [37]. Similarly, the quasi-inelastic peak appearing in neutron spectra (see figs. 2 and 3 ) is due to a first collision corresponding to $p p \rightarrow n \Delta^{++}$or to $p n \rightarrow n \Delta^{+}$with the neutron emitted at forward angles. There are also minor contributions coming from $\Delta$ 's emitted in the forward directions. As barely seen from figs. 1 and 2 and more clearly from fig. 3 and from ref. [9], the INCL model reproduces these quasi-inelastic peaks rather crudely, missing in particular the shape of the peaks.

The predictions of INCL for the quasi-elastic and quasi-inelastic peaks contrast with those for the rest of the spectra above $\sim 20 \mathrm{MeV}$, corresponding to emission of the detected particles issued from second, third, etc., collisions. This illustrates the paradoxical performances of INC models mentioned above. 


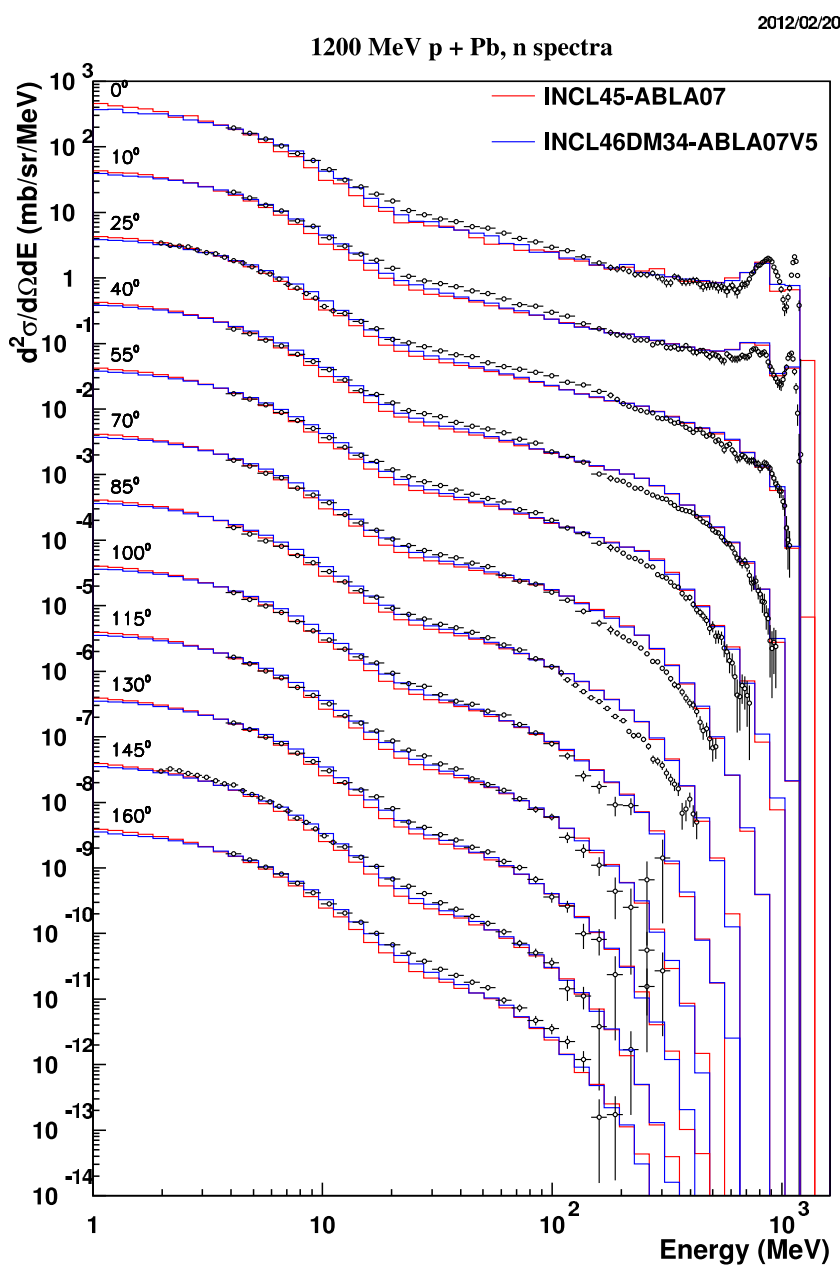

Fig. 2. Neutron double differential cross sections for $p(1200 \mathrm{MeV})+{ }^{208} \mathrm{~Pb}$ reactions, as functions of the neutron kinetic energy and at different angles, indicated on the figure. For the sake of clarity, the spectra at the various angles have been multiplied by $10^{0}, 10^{-1}, 10^{-2}$, etc, for successive angles in increasing order. Data (symbols) are from ref. [36]. They are compared with the predictions of INCL4.5 (red histograms) and INCL4.6 (blue histograms). For more detail, see ref. [24].

\subsection{Coherent excitation of the target nucleus and coherent transfer reactions}

Here we are interested in the high-energy part of proton (or neutron) spectra in $(p, p x n)$ or $(n, x n)$ reactions or in transfer reactions. Figure 4 gives an example of a $(n, x n)$ reaction. A characteristic feature of these spectra is the presence of a generally strong high energy part (typically the highest $10 \mathrm{MeV}$ or so) contrasting with the rest of the spectra which is smoothly varying with the energy of the detected particle. This high-energy part is generally attributed to the excitation of the target to low-lying excited states. In some data, at lower incident energy, the excited states are resolved, whereas in the case of fig. 4, their contribution appears as a more or less structureless bump. Note that this contribution is visible at rather low incident energy. For large incident energy (like in fig. 2), the contribution is located above the quasi-elastic peak (in neutron energy) and restricted to very small angles. In addition, the absolute value of the cross section is smaller than at low incident energy. It is thus not visible on the scale of fig. 2.

In such reactions, leading to the excitation of low-lying states, the target is very little disturbed and acts almost coherently. Contrarily to the other phenomena that we discussed, the reaction cannot be attributed to a single scattering, but nevertheless should imply very few (possibly collective) degrees of freedom. They dominantly correspond to $(p, n)$ or $\left(n, n^{\prime}\right)$ reactions, with the emission of only one neutron. Theses considerations are also valid for transfer reactions like $(d, x n)$ reactions [38].

Figure 4 also shows that INCL cannot reproduce the high-energy part of the spectra, but reproduces fairly well the rest of the spectra, expectedly resulting to incoherent processes akin to the cascade collision dynamics.

We will not discuss these reactions further in this paper. But we want to emphasize a recent development, which is described in ref. [39]. It almost nearly consists in taking the mean of the incoherent cross sections implied by INCL4 and of the coherent low-energy excitations, calculated by DWBA method. We give an illustrative result in fig. 5 . Although the agreement is not perfect, the figure clearly shows that INCL is rather appropriate for neutron energy below $35 \mathrm{MeV}$ 


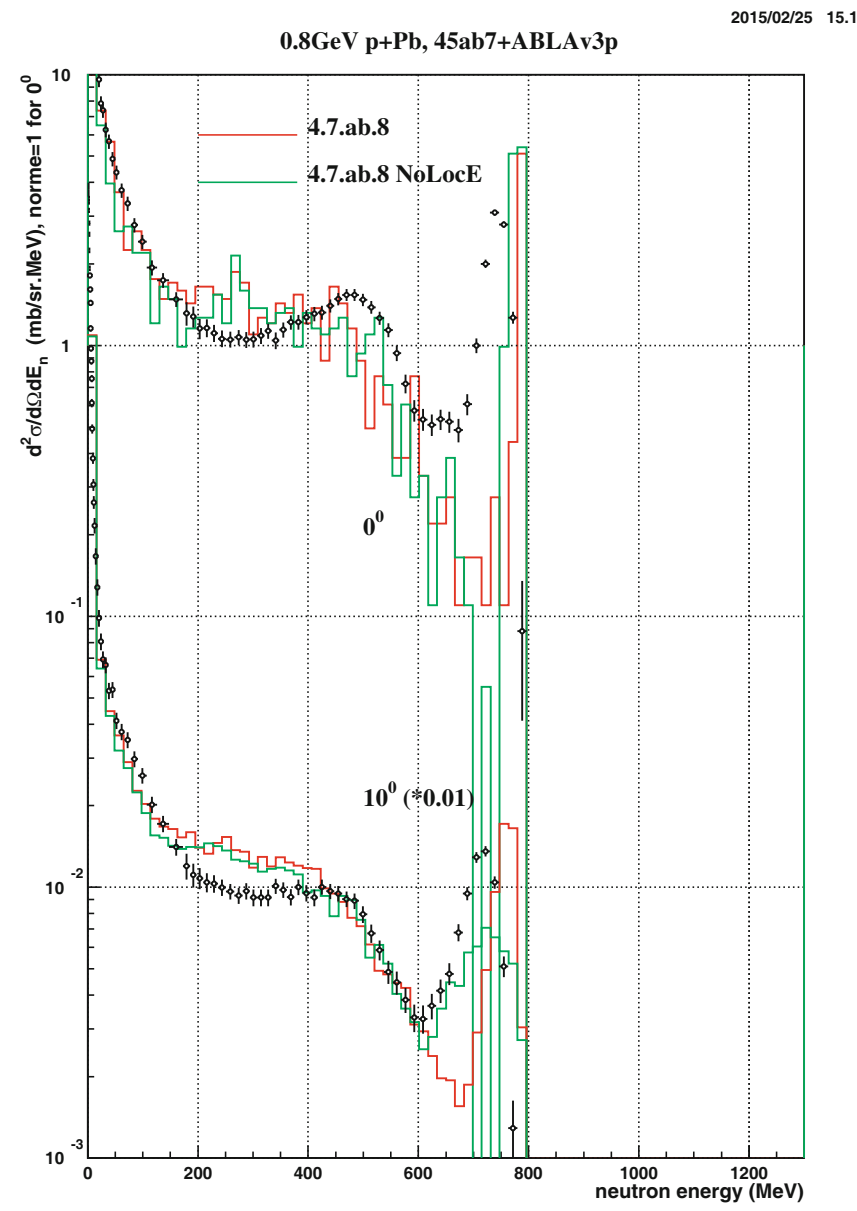

Fig. 3. Neutron double differential cross sections $\mathrm{d} \sigma / \mathrm{d} \Omega \mathrm{d} E_{n}$ for $p(800 \mathrm{MeV})+{ }^{208} \mathrm{~Pb}$ reactions, as functions of the neutron kinetic energy $E_{n}$, at two angles indicated on the figure. For the sake of clarity, the spectrum at ten degrees has been multiplied by 0.01. Data (symbols) are from ref. [36]. They are compared with the predictions of INCL4.6 (red histograms). The green histograms correspond to the predictions of INCL4.6 when the so-called LocE recipe is removed (see sect. 5 below). For more detail, see text and ref. [24].

(giving a hint for the validity of INCL and the collision picture for this part of the reaction process), whereas the bulk of the cross sections for larger neutron energy is dominated by coherent excitations described by DWBA. This result is very encouraging. Further work is needed to test the generality of this approach and to understand why a simple mean of the two contributions seems to be sufficient.

\subsection{Production of near-target residues}

We now turn our interest to cross sections for the production of nuclides close to the target, i.e. with similar $A$ and $Z$. These cross sections are typically associated with few-collision reactions, but the latter are not directly corresponding to exclusive cross sections. The requirement of producing a particular nuclide typically imposes strict constraints on the collision outcome. We are going to review these constraints for the production of isotopes ${ }^{\prime} A^{\prime}$ residues with $Z^{\prime}=Z+1, Z, Z-1$ and $A^{\prime}=A+1, A, A-1$.

\subsubsection{One-nucleon removal cross sections}

At first sight, the removal of one nucleon leading to the production of ${ }^{A-1}(Z-1)$ and ${ }^{A-1} Z$ residues in $p+{ }^{Z} A$ reactions, corresponding to one proton and one neutron removal, respectively, should result from a single proton-proton or a single proton-neutron collision respectively. Equivalently, they correspond to $(p, 2 p)$ and $(p, p n)$ reactions. Even in the context of cascade+evaporation models, the mechanism for removal of one nucleon should be precised a little bit. 


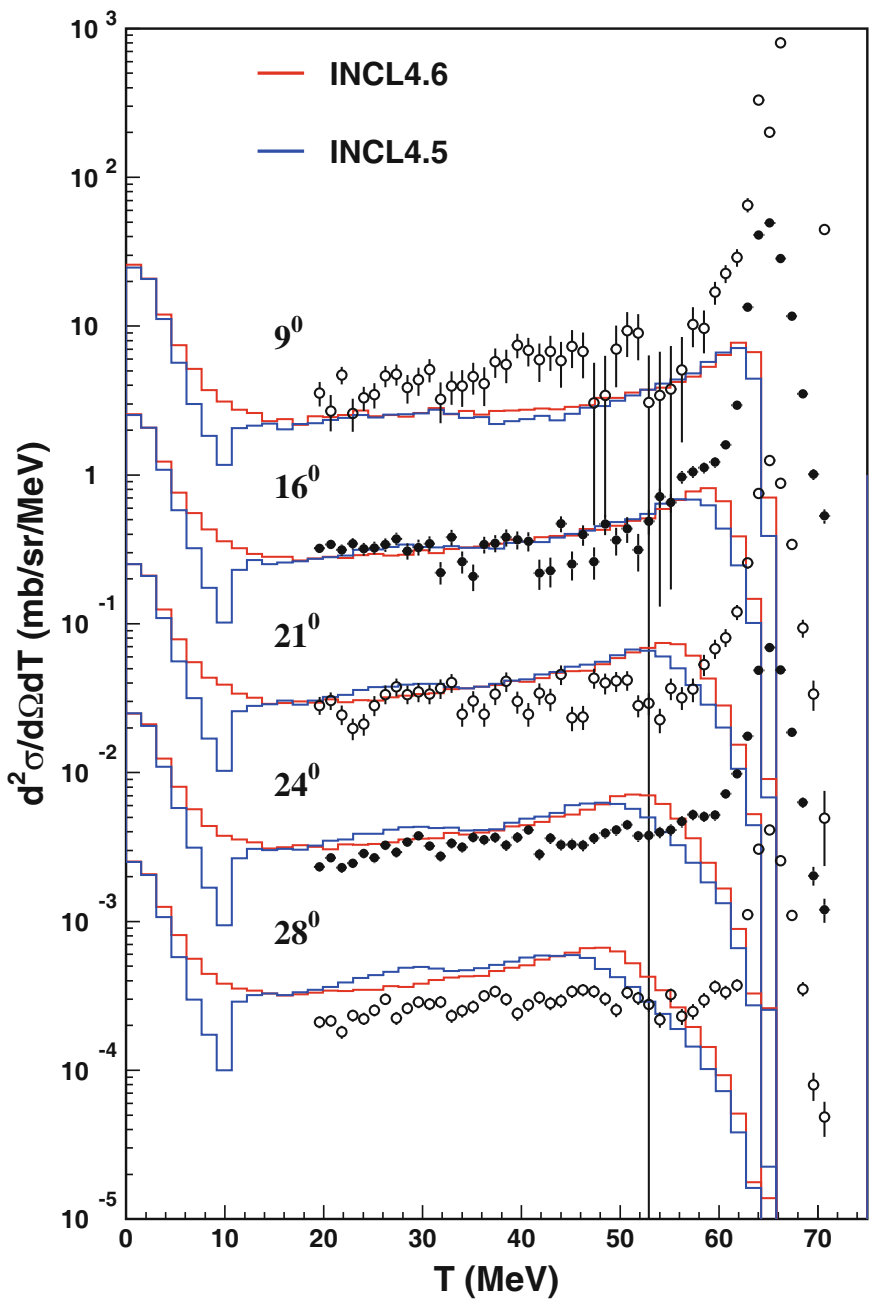

Fig. 4. Inclusive neutron double differential cross section for the $n(65 \mathrm{MeV})+{ }^{\text {nat }} \mathrm{Fe}$ reaction. Neutrons are detected at various angles indicated on the figure. Experimental data [40] are represented by small circles. For the sake of clarity, the spectra at the various angles have been multiplied by $10^{0}, 10^{-1}, 10^{-2}$, etc., for successive angles in increasing order. Theoretical results calculated with the INCL4.6 [24] plus ABLA07 [27] model, are denoted by red histograms. More details in the text.

Strictly speaking, in the case of one-proton removal, there should be only one single elastic $p p$ collision leading to two protons which do not interact any more and to a remnant with an excitation energy which is below the lowest particle emission threshold (generally the neutron threshold). In principle, one should not exclude additional collisions made by the protons on their way out, nor further collisions made by the remnant nucleons. But, these collisions should by very, very soft, otherwise a small additional excitation energy of the target remnant might lead easily to the evaporation of a neutron, moving so the system outside the $(p, 2 p)$ channel. Moreover, these soft collisions should be largely Pauli-blocked. We indeed checked that it is so in the INCL model, the $(p, 2 p)$ channel being overwhelmingly dominated by one-collision events. In the rest of this paper, in order to simplify the vocabulary, we will deliberately forget about mentioning these soft collisions.

Also, it should be mentioned, that, if the incident energy is sufficiently large, the removal of one proton may result from an inelastic $p p$ collision leading to the production of a $\Delta$ resonance $\left(p p \rightarrow p \Delta^{+}\right)$. In such a case, the $\Delta^{+}$should decay in a proton and a $\pi^{0}$ without bringing to the remnant an excitation energy larger than the neutron separation energy. Furthermore, the three involved particles should be able to basically go out of the target without further interaction ${ }^{4}$.

\footnotetext{
${ }^{4}$ Actually, one-nucleon removal cross section may not only involve with the exclusive $(p, N N)$ and $(p, N N \pi)$ processes, since, for instance, $(p, p n x \gamma)$ reactions contribute to the one-proton removal cross sections. This latter contribution is likely rather small, except for some specific residues. We disregard them in this discussion.
} 


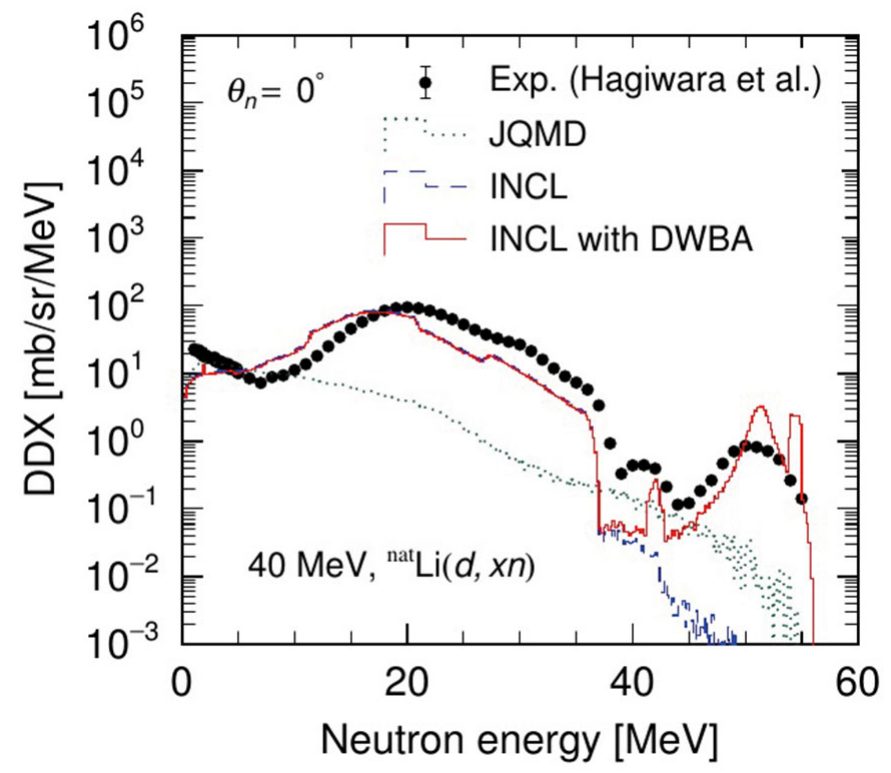

Fig. 5. Double differential neutron production cross section at emission angle $\theta_{n}=0$ in ${ }^{\text {nat }} \operatorname{Li}(d, x n)$ for $40 \mathrm{MeV}$ incident deuterons. Experimental data (heavy dots), from ref. [41], are compared with calculated results obtained by INCL alone (blue line) and by the INCL-DWBA combination (red lines). The finely dotted line corresponds to the predictions of the JAERI Quantum Molecular Dynamics (JQMD) model [42]. The blue and red lines are superposed for neutron energy below $\sim 36 \mathrm{MeV}$. See ref. [39] for detail.

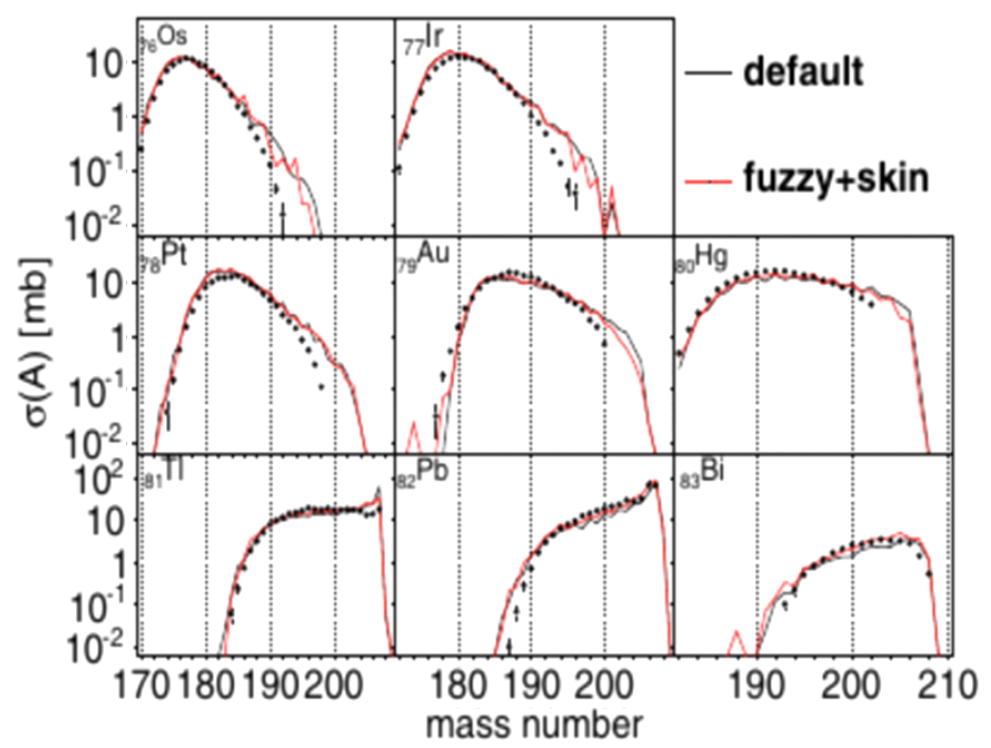

Fig. 6. Isotopic distributions of the $Z=76-83$ residues produced in $p(1 \mathrm{GeV})+{ }^{208} \mathrm{~Pb}$ reactions. The standard INCL4.6+ABLA07 calculation (black lines) is compared to the experimental data of refs. [43,44] (dots) and to the refined calculation (red lines) described below (see sect. 5).

Similarly, for the production of ${ }^{Z}(A-1)$ residues (removal of one neutron), one should consider mechanisms involving either a single $(p n)$ elastic collision or a single inelastic $p n \rightarrow p \Delta^{0}$, followed by $\Delta^{0} \rightarrow n+\pi^{0}$, or still a single inelastic $p p \rightarrow p \Delta^{+}$collision, followed by $\Delta^{+} \rightarrow n+\pi^{+}$, with the condition of small enough excitation energy of the remnant. In addition, the particles involved in the mechanisms described above should not make other collisions than the ones corresponding to these mechanisms.

In addition, in the $(p, p n)$ case, one should also consider the following mechanism: the incident proton hits a proton or a neutron, leaves the target nucleus without further collision and the struck target nucleon brings to the remnant an excitation energy larger than the neutron threshold and lower than the two-neutron emission threshold. In that case, the emitted neutron is issued from the evaporation. 

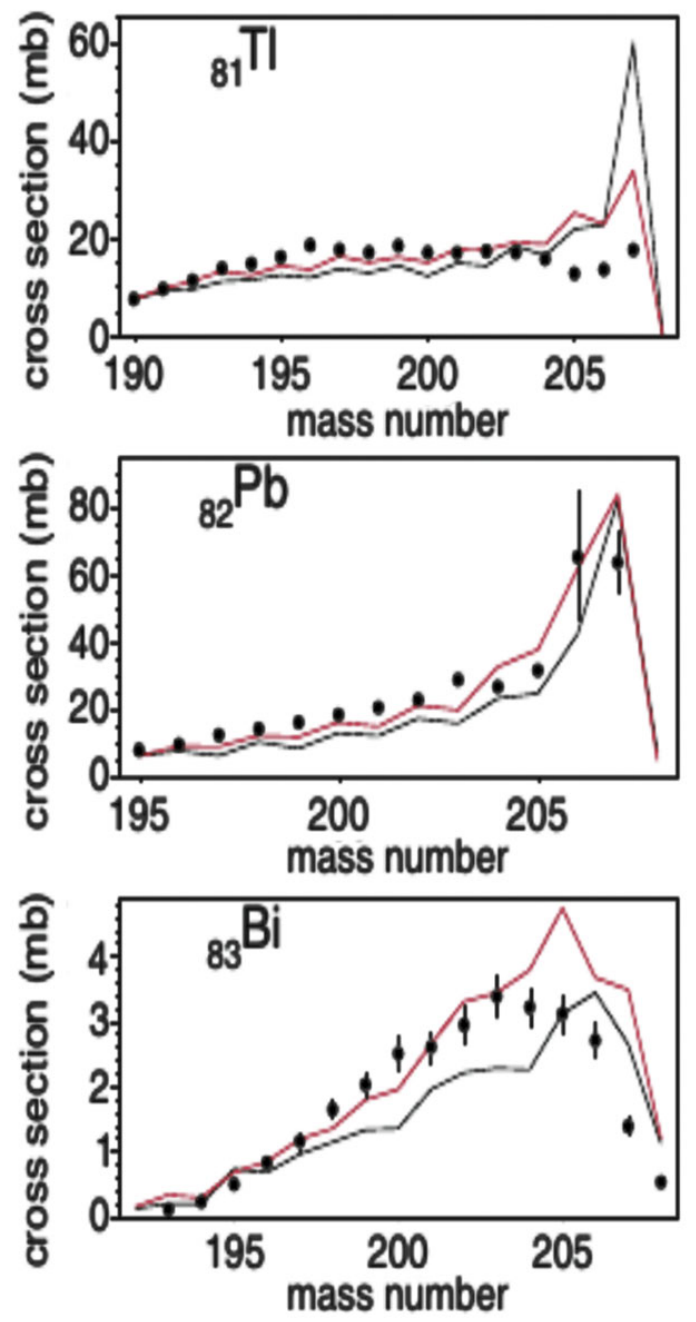

Fig. 7. Same as fig. 6 for the $Z=81-83$ residues, with a linear scale.

We now want to discuss how the INCL model describes these one-nucleon removal cross sections. It is perhaps interesting to first look at fig. 6 which gives an overview of several isotopes neighbouring the target nucleus. One can realize that there is a globally satisfactory agreement between the predictions of INCL and the data. Actually, it appears that a clear disagreement exists for the heaviest isotopes with $Z \leq 80$. However, these cross sections are generally smaller than $1 \mathrm{mb}$. Thus these is a real global agreement for large cross sections.

The discrepancy for production of isotopes very close to the target which we alluded to above can be more easily seen in fig. 7. The striking feature here is the strong overestimation by INCL of the ${ }^{207} \mathrm{Tl}$ production cross section. This is the isotope reached through the $(p, 2 p)$ channel. The production of the ${ }^{207} \mathrm{~Pb}$ isotope, corresponding to the $(p, p n)$ channel, is also slightly overestimated.

The general character of the results for the $(p, 2 p)$ and $(p, p n)$ channels is illustrated by fig. 8. As a matter of fact, in first approximation, the experimental cross sections for both channels do not seem to vary much with the target mass or with the incident energy. In second approximation the $(p, 2 p)$ cross section is slightly larger for light targets. The experimental $(p, p n)$ cross section shows the same trends. It is systematically larger that the $(p, 2 p)$ cross section. More importantly, the situation contrasts with the theoretical calculations of INCL4.6 which predicts a $(p, p n)$ cross section roughly equal to the $(p, 2 p)$ one. This is more or less expected in view of the near equality between the elementary $p p$ and $p n$ cross sections in the range of the incident energy. As a result the predicted $(p, 2 p)$ cross section is overestimated by roughly a factor 2 , whereas the $(p, p n)$ cross section comes roughly in agreement with the experimental data. The same tendency occurs also in the results of other cascade-evaporation models, although, sometimes, details may be quite different. See ref. [26] for a discussion of this point. 
$(\mathrm{p}, 2 \mathrm{p})$

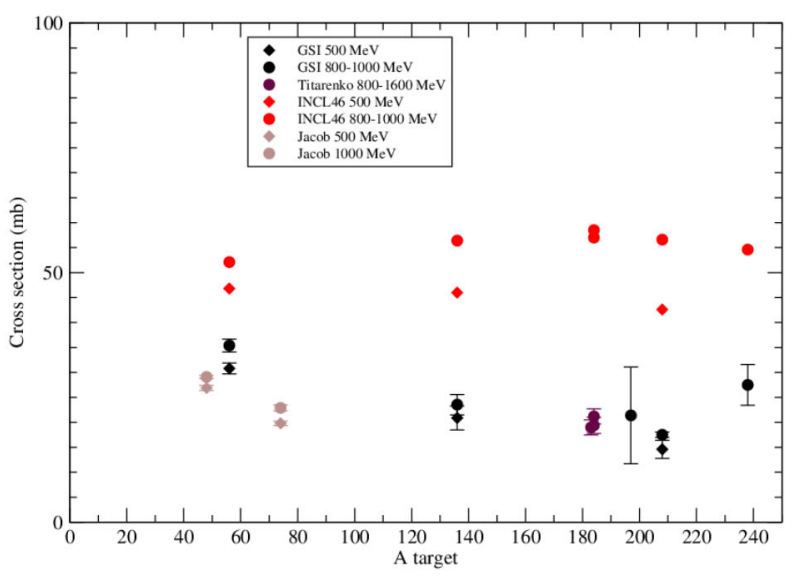

(p,pn)

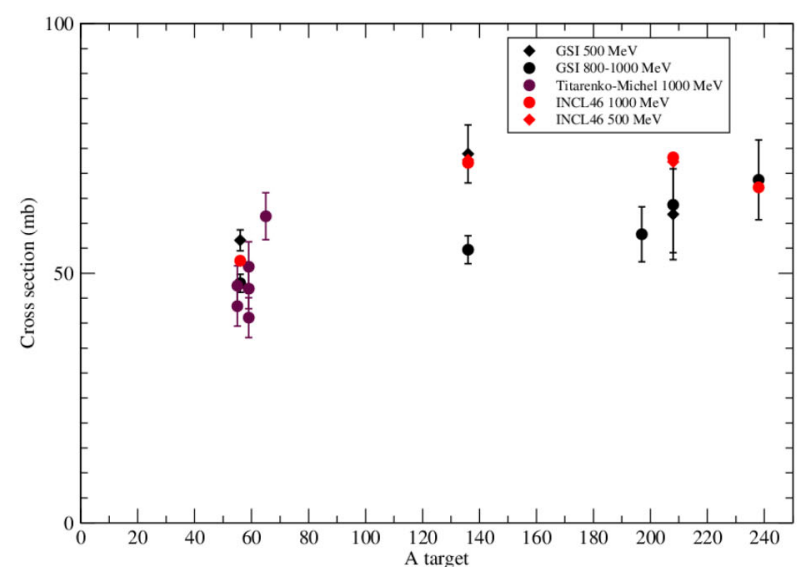

Fig. 8. Production cross sections of ${ }^{A-1}(Z-1)$ isotopes $\left((p, 2 p)\right.$ reaction, left panel) and of ${ }^{A-1} Z$ isotopes $((p, p n)$ reaction, right panel) for several ${ }^{A} Z$ targets and at various proton incident kinetic energies, indicated on the panels. The data (black symbols), taken from refs. [43,45-55], are compared with the theoretical predictions of INCL4.6+ABLA07 (red symbols).

\subsubsection{The production of $\mathrm{Z}+1$ residues}

Here, the most singular case is the production of ${ }^{Z+1}(A+1)$ residues, corresponding to the $\left(p, \pi^{0}\right)$ reaction. In terms of collisions (in models where pions are produced via $\Delta$-resonances, as in INCL), this covers the following possibilities:

1) $p p \rightarrow p \Delta^{+}, \Delta^{+} \rightarrow p \pi^{0}$ when the two protons are stopped in the target nucleus and deliver to the latter a total excitation energy lower than the neutron emission threshold and when the pion escapes any further interaction.

2) $p n \rightarrow p \Delta^{0}, \Delta^{0} \rightarrow n \pi^{0}$ with similar constraints.

3) $p n \rightarrow n \Delta^{+}, \Delta^{+} \rightarrow p \pi^{0}$ with similar constraints.

Such mechanisms seem to be very unlikely. In fact, they are. In the first one, for example, if the target proton was at rest, it would receive a sizeable energy transfer, even if it is produced in the forward direction. It is only when this target nucleon has a momentum close to the Fermi momentum and a direction opposite to the incoming nucleon that it has a chance to be produced with a final momentum which is just above the Fermi momentum. Similarly, the proton issued from the $\Delta$-resonance should be emitted at special angles in order to have a final momentum close to the Fermi momentum. These special conditions are at the origin of the very small production cross section, as analyzed in detail in ref. [56].

To emphasize the very special character of the mechanism it is sufficient to notice that almost all the incident momentum should be carried out by the pion while only a small fraction of the incident energy minus the pion rest mass energy is transferred as excitation energy to the target nucleus. This, of course, requires rather stringent conditions.

In fig. 9, we show the predictions of INCL4 for the production of ${ }^{210} \mathrm{Po}$ in $p+{ }^{209} \mathrm{Bi}$ reaction. Actually, predictions are shown for several versions of INCL. Basically the predictions of INCL4.2 [19] are represented by the dotted curves and those of INCL4.3 [58] by the red curve. The predictions of later versions INCL4.5 [20] and INCL4.6 [24] are very similar to those of INCL4.3. See refs. $[58,24]$ for a discussion of the various versions.

One has to notice first that the cross section is very small, about $5-8 \mu \mathrm{b}$. It is roughly reproduced by the INCL4.3 and later versions of INCL. This indicates that the INCL models pick up the right production mechanism even if this production occurs close to the boundary of the available phase space.

The production of ${ }^{Z+1} A$ residues is possible through the $(p, n),\left(p, n \pi^{0}\right)$ and $\left(p, p \pi^{-}\right)$channels. In terms of mechanisms based on collisions, they correspond to the following schemes:

1) $p n \rightarrow p n$ where the proton outgoing from the collision has a very small energy, so small that it can be stuck in the target nucleus, and the final neutron leaves the remnant basically undisturbed. This is favoured by the backward (or exchange) scattering when the neutron is emitted in the direction of the incoming proton. By the term stuck, we do not mean that a particle (here basically a nucleon) is stopped in the target by successive collisions. Rather, we mean that the nucleon issued from a nucleon-nucleon collision is eventually left in the target remnant with an energy below the nucleon emission threshold and that the excitation energy of the remnant $E^{*}$ is smaller than the lowest particle emission threshold in evaporation $S_{\min }$, usually the neutron emission treshold ${ }^{5}$. In INCL models, this

${ }^{5}$ Even if not stated, the term "stuck" means $E^{*} \leq S_{\text {min }}$. 


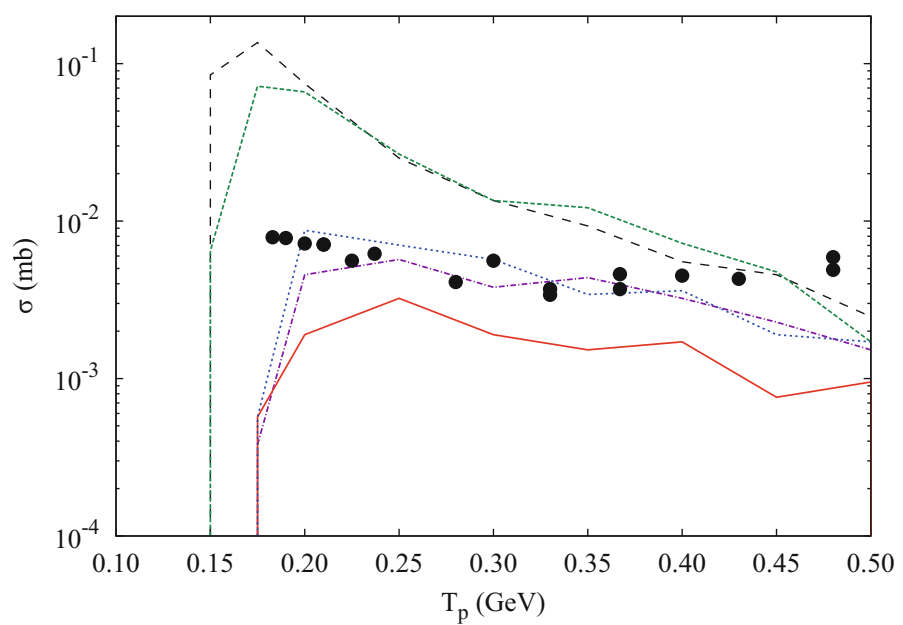

Fig. 9. Cross section for production of ${ }^{210} \mathrm{Po}$ isotopes in $p+{ }^{209} \mathrm{Bi}$ reactions, as functions of the kinetic energy $T_{p}$ of the incident proton. Black symbols correspond to the experimental data of ref. [57], after removal of the $(p, \gamma)$ background. The theoretical curves correspond to various versions of the INCL4 model, with the 4.2 version in black dashes and the 4.3 version in red. This figure is inspired from fig. 3 of ref. [56].

basically requires that the "stuck" nucleon is produced with a low kinetic energy, being so trapped in its potential well. Along with the warning expressed in sect. 3.3.1, possible very soft collisions undergone by this nucleon may not be excluded, but their importance, if they are not simply Pauli-blocked, is very minute. In order to simplify our presentation of similar cases, we simply exclude this possibility from our comments.

2) $p n \rightarrow n \Delta^{+}, \Delta^{+} \rightarrow p \pi^{0}$ with the conditions that the neutron outgoing of the collision leaves the target undisturbed and that the proton issued from the $\Delta$ has very small energy and gets stuck in the target and finally that the pion emerges undisturbed from the target nucleus.

3) $p n \rightarrow p \Delta^{0}, \Delta^{0} \rightarrow p \pi^{-}$, when one of the protons gets stuck in the target and when the other proton and the pion are escaping.

4) $p n \rightarrow p \Delta^{0}, \Delta^{0} \rightarrow n \pi^{0}$, when the proton gets stuck in the target and when the neutron and the pion are escaping.

These machanisms are very close to those that have been encountered for quasi-elastic and quasi-inelastic scatterings (see sect. 3.1), except that for the former only one neutron is required to leave the target whereas for the latter there is no constraint of this sort.

In fig. 7 , it is shown that the production cross section for a ${ }^{Z+1} A$ residue (here the production of ${ }^{208} \mathrm{Bi}$ in $p(1 \mathrm{GeV})+$ ${ }^{208} \mathrm{~Pb}$ reactions) is small, of the order of one millibarn. See the lower panel of the figure. Here also the INCL model satisfactorily describes the order of magnitude of the cross section.

The production of ${ }^{Z+1}(A-x)$ residues, with $x \geq 1$ involves more than a collision and, as $x$ increases, the evaporation of neutrons is more and more probable. Typical results for INCL are displayed in fig. 7.

\subsubsection{The production of $\mathrm{Z}+2$ residues}

For these residues, the most singular case is the production of ${ }^{Z+2}(A+1)$ residues, corresponding to the $\left(p, \pi^{-}\right)$ channel. In terms of collisions, this covers the following mechanism: $p n \rightarrow p \Delta^{0}, \Delta^{0} \rightarrow p \pi^{-}$, subject to the condition that both protons are produced with low energy and are stuck in the target. As for the ${ }^{2}+2(A-x)$ residues, with $x \geq 0$, the possible production mechanisms involve more than one collision and emit neutrons that can be produced more and more by evaporation, as $x$ increases. We will come back to the production of $Z+2$ residues in the following.

The comparison between the predictions of INCL4 and the experimental data is displayed in fig. 10, for the particular case of the production of Astatine isotopes in $p(800 \mathrm{MeV})+{ }^{209} \mathrm{Bi}$ reactions. The heaviest possible isotope corresponds to $Z+2$ and $A+1$, with respect to the target. One can notice that the cross section for this isotope (not visible in fig. 10), is very small, less than one $\mu \mathrm{b}$, as in the case of sect. 3.3.2. It can also be seen that the predictions of INCL catch the right order of magnitude, but are not fully satisfactory.

Similar results have been obtained [60] for the production of $Z=56$ (baryum) isotopes in $p(1 \mathrm{GeV})+{ }_{54}^{136} \mathrm{Xe}$ reactions, as measured in ref. [50]. 


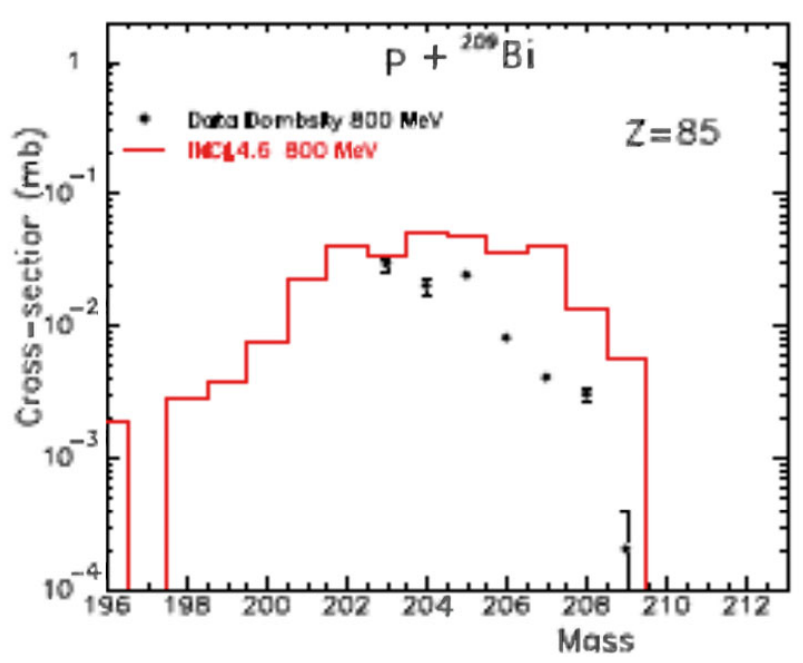

Fig. 10. Cross section for production of astatine $(Z=85)$ isotopes in $p(800 \mathrm{MeV})+{ }^{209} \mathrm{Bi}$ reactions, as a function of their mass number. Black symbols correspond to the experimental data of ref. [59]. The red curve corresponds to the results of the INCL4.6 plus ABLA07 model [24]. This figure is inspired from fig. 7 of ref. [60].

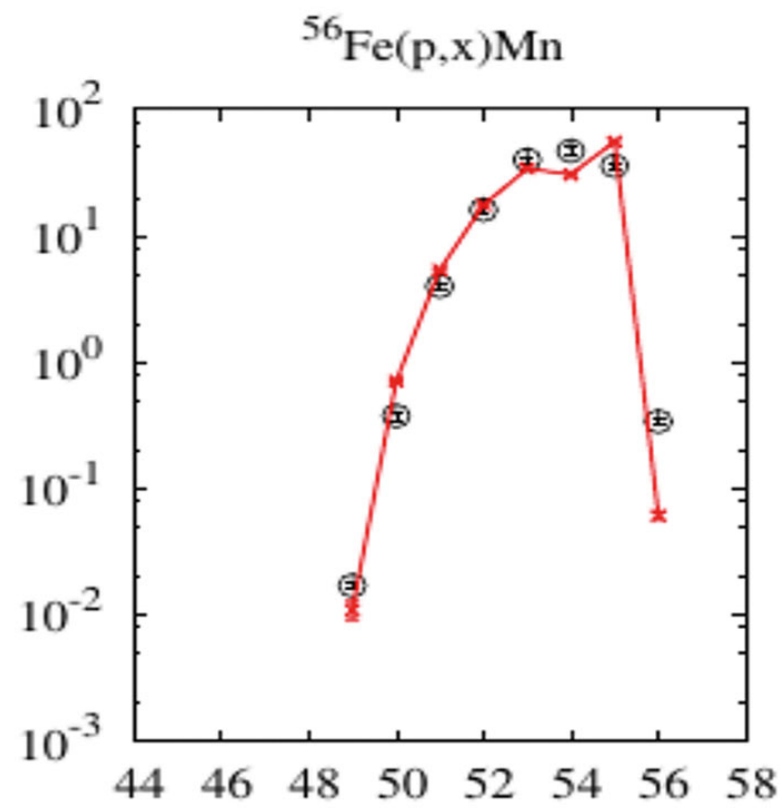

Fig. 11. Cross section (in mb) for production of manganese $(Z=25)$ isotopes in $p(1 \mathrm{GeV})+{ }^{56}$ Fe reactions, as a function of their mass number. Black symbols correspond to the experimental data of ref. [47]. The theoretical curve (in red) corresponds to predictions of INCL4.5+ABLA07. More detail in refs. [20,9].

\subsubsection{The production of Z-1 residues}

The heaviest isotope of this sort that can be produced is ${ }^{Z-1} A$. This reaction proceeds through the $\left(p, p \pi^{+}\right)$channel, corresponding either to the $p p \rightarrow p \Delta^{+}, \Delta^{+} \rightarrow n \pi^{+}$mechanism, or to the $p p \rightarrow n \Delta^{++}, \Delta^{++} \rightarrow p \pi^{+}$mechanism, both subject to the condition that the neutron gets stuck in the target and that the pion and the proton emerge undisturbed. The production of this isotope has not been measured very often. The data for the example of the production of $M n$ isotopes in $p(1 \mathrm{GeV})+{ }^{56} \mathrm{Fe}$ reactions are shown in fig. 11. The numerical value of the cross section for the production of ${ }^{56} \mathrm{Mn}$ lies around half a mb. The order of magnitude is reproduced by the INCL4 model.

The production of the second heaviest isotope $(Z-1(A-1))$ is of course related to the exclusive $(p, 2 p)$ reaction discussed in sect. 3.3.1. The production of these residues may be due to a single $p p$ elastic collision, but may also be due to a single inelastic scattering $p p \rightarrow p \Delta^{+}$, followed by $\Delta^{+} \rightarrow n \pi^{+}$(or $\Delta^{+} \rightarrow p \pi^{o}$ ), provided the two nucleons and the pion leave the target remnant with an excitation energy smaller that the neutron emission threshold energy. 


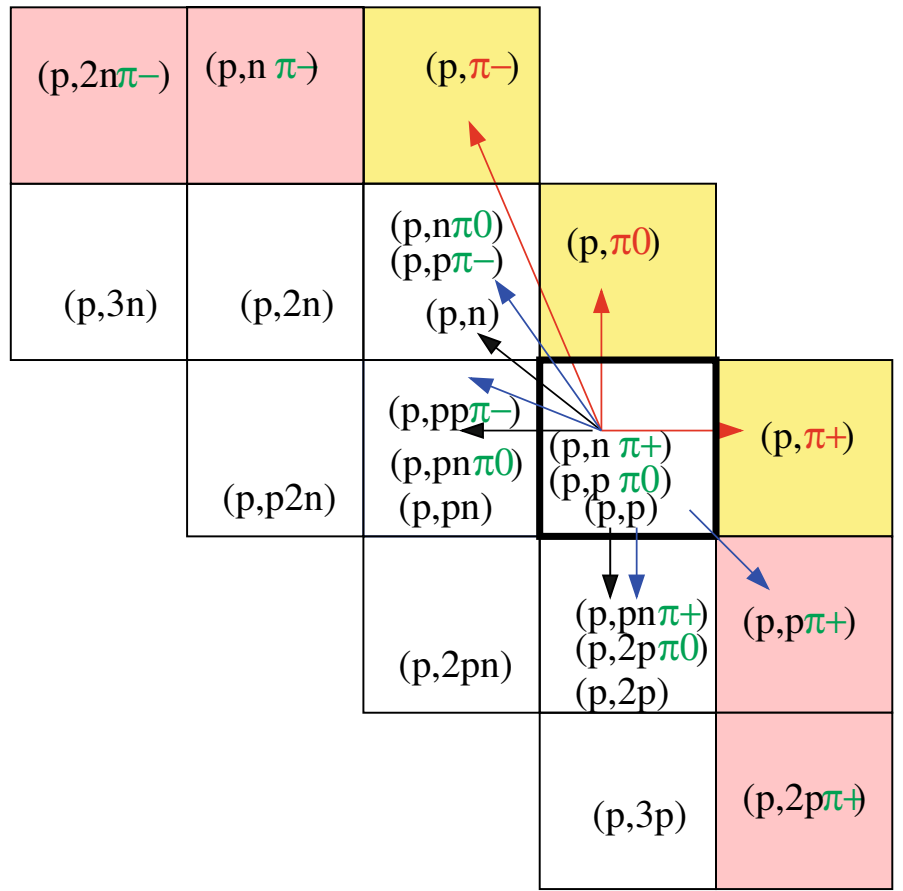

Fig. 12. Schematic representation in the $N-Z$ plane of the residues neighbouring the target nucleus ${ }^{A} Z$ (corresponding to the square with the bold contour) with indications of the main reactions leading to these residues. Coloured squares correspond to residues that can be produced owing to pion production only. $A+1$ residues are depicted by the yellow squares. Pink squares correspond to other $Z+2$ (top) and $N+1$ (right) residues. Red arrows indicate the isotopes which are accessible through $(N, \pi)$ channels only. Black arrows mean that the corresponding isotopes can be formed through mechanisms involving one elastic collision. Blue arrows indicate that the corresponding isotopes can be formed in mechanisms involving one single inelastic collision. Note that the square representing at the same time the target nucleus and the residue with same $N$ and $Z$ should be considered as implicitly connected by both a black and a blue arrows. See text for more detail. This figure is inspired from fig. 1 of ref. [56], in which small mistakes have been corrected.

The production of ${ }^{Z-1}(A-x)$ residues with $x \geq 2$ corresponds, as above, to a reaction mechanism with at least two collisions and involving more and more evaporation of neutrons as $x$ is increasing.

\subsubsection{The production of $Z$ residues}

Similarly to the other cases discussed above, the heaviest produced isotope is ${ }^{Z}(A+1)$, through the $\left(p, \pi^{+}\right)$channel, owing to the $p p \rightarrow p \Delta^{+}, \Delta^{+} \rightarrow n \pi^{+}$(with the condition that both nucleons get stuck in the target) and $p n \rightarrow n \Delta^{+}$, $\Delta^{+} \rightarrow n \pi^{+}$(with similar constraints) mechanisms. The ${ }^{Z} A$ isotope is produced, aside to the coherent elastic protonnucleus scattering, through the $p p \rightarrow p p$ and $p n \rightarrow p n$ channels, with the condition that a proton or a neutron (resp.) gets stuck in the target. This case is, of course, similar to the production of the ${ }^{Z+1} A$ isotope discussed in sect. 3.3.2. Thus the pion channels should be considered as well.

The $^{Z}(A-1)$ isotope is produced through the $(p, p n)$ channel, involving a reaction mechanism in which the incident proton collides elastically with a target neutron and the two outgoing particles leave the target undisturbed. Of course, as already discussed in sect. 3.3.1, pion channels are also contributing to the production of this isotope (see fig. 12 below).

From fig. 7, it can be seen that the theoretical prediction of the cross section for the production of the ${ }^{Z} A$ isotope indicates a small cross section of a few mb. As already mentioned the experimental cross section for the production of ${ }^{Z}(A-1)$ is large and it is slightly overestimated by the INCL model.

\subsubsection{Overview}

The discussion above can be summarized in a more systematic way using fig. 12, which represents the location of the residues close to the ${ }^{A} Z$ target nucleus in the $N-Z$ plane. The target nucleus is symbolized by the square with the bold contour. The considerations made in this sect. 3.3 can be reformulated as follows. 
1) The $A+1$ isotopes represented by the yellow boxes can be formed through a single inelastic $N N \rightarrow N \Delta$ collision, followed, of course, by the decay of the produced $\Delta$, subject to the condition that the two involved nucleons (the free one and the one coming from the $\Delta$ decay) should remain stuck in the target. In addition, the pion should leave the nucleus undisturbed. These isotopes cannot be formed by a single elastic collision.

2) Concerning the $A$ isotopes one has to distinguish between the cases. The $Z+2$ isotope cannot be formed by a single collision. The $Z-1$ isotope can be made through a single inelastic collision. The two remaining isotopes, corresponding to the two white $A$ boxes, can be reached through either an elastic or an inelastic collison. For these isotopes and the preceding $Z-1$ one, the mechanism is subject to the condition that one of the involved nucleons should be stuck in the target nucleus and that the other should leave the target undisturbed, as well as the possible pion.

3) As for the $A-1$ isotopes, one can easily show that the $Z+1$ isotope (box with $(p, 2 n)$ indication) and the $Z-2$ isotope (box with $\left(p, 2 p \pi^{+}\right)$indication) cannot be produced through a mechanism involving a single collision. The remaining $Z$ and $Z-1$ isotopes can be made through a mechanism involving a single elastic or inelastic collision, provided that all the particles emerging from the collision can leave the nucleus unperturbed and that the residual excitation energy of the latter is smaller than the neutron emission threshold energy. This is, of course, in line with the discussion of one-nucleon removal cross sections in sect. 3.3.1.

To complete a little bit the comment about fig. 12, one can mention that isotopes with mass number larger than $A+1$ (on the right of the line of the yellow squares) cannot be produced in any case. Isotopes with $Z+3$ charge can be produced in $(p, 2 \pi)$ channels, which obviously involves at least two collisions (at least in INCL ${ }^{6}$ ). Similarly, isotopes with neutron number larger than $N+1$ (on the right of the vertical pink "line") can only be formed through production of more than one pion, thus involving at least two collisions. The isotopes that can be formed through mechanisms involving a single collision are only those corresponding to the squares in fig. 12 that are connected to the target nucleus square by arrows, including the target nucleus itself. For all the other isotopes, the possible mechanisms of formation involve either several collisions or evaporation, of neutrons mainly.

One has to notice that the pattern of reactions is not symmetric with respect to the main diagonal in fig. 12 passing through the target box. This is, of course, due to the fact that we consider only proton as a projectile.

\subsection{Recoil velocity of ${ }^{A}(Z+1)$ residue}

As we have seen in sect. 3.3.6, the production of this isotope can proceed through a single $p n$ elastic collision, provided the neutron escapes further collision and that the proton remains stuck in the target and delivers a very small excitation energy. The production of this isotope can also proceed through an inelastic $p n \rightarrow p \Delta^{0}$ collision, followed by the $\Delta^{0} \rightarrow n \pi^{0}$. In that case, the neutron and the pion should leave the nucleus without further collision and the proton should remain stuck in the target, transferring a small excitation energy. To be complete, the reaction can also proceed through the sequence $p n \rightarrow n \Delta^{+}, \Delta^{+} \rightarrow p \pi^{0}$ subject to similar conditions.

Recoil velocity distributions have been measured at GSI for many residues, in reverse kinematics. The recoil velocity of the residue in direct kinematics thus appears, in reverse kinematics, as the difference of the heavy nucleus incident velocity and the residue velocity. Actually, only the longitudinal component is measured. Figure 13 shows the example of the velocity distribution of the ${ }^{208} \mathrm{Bi}$ residues in ${ }^{208} \mathrm{~Pb}+p$ and ${ }^{208} \mathrm{~Pb}+d$ reactions with $1 \mathrm{GeV}$ per nucleon ${ }^{208} \mathrm{~Pb}$ projectiles.

The velocity indicated in fig. 13 is most often negative, because the incident nucleus is slowed down a little bit in the process of residue formation. It is opposite to the recoil velocity that the residue would get in direct kinematics measurements. We will use the "direct kinematics vocabulary" hereafter.

For the $p+{ }^{A} Z \rightarrow n+{ }^{A}(Z+1)$ reaction, energy-momentum conservation enforces a one-to-one correspondance between the longitudinal recoil velocity of the residue and its excitation energy, at zero degree at least. Indeed, in the ${ }^{A} Z$ frame, one has the following conservation laws when the neutron (and the ${ }^{A}(Z+1)$ residue) is emitted in the forward direction:

$$
\begin{aligned}
p_{0} & =p_{R}+p_{1} \\
\sqrt{p_{0}^{2}+m^{2}}+M & =\sqrt{p_{R}^{2}+M_{R}^{2}}+\sqrt{p_{1}^{2}+m^{2}}
\end{aligned}
$$

In these equations, $p_{0}, p_{R}$ and $p_{1}$ are the momenta of the incident proton, of the recoiling residue and of the emitted neutron, respectively. The quantities $m, M$ and $M_{R}$ are the masses of the proton, of the target and of the possibly excited residue, respectively. Eliminating $p_{1}$ from eqs. (4) and (5), and writing $M_{R}=M-Q+E^{*}$, where $Q$ is the $Q$-value of the reaction, and $E^{*}$ the residue excitation energy, one gets an equation relating implicitly $E *$ to $p_{R}$.

\footnotetext{
${ }^{6}$ In reality, two pions may be issued from a nucleon-nucleon collision. However, the corresponding cross section is very small at the energies involved in this paper.
} 


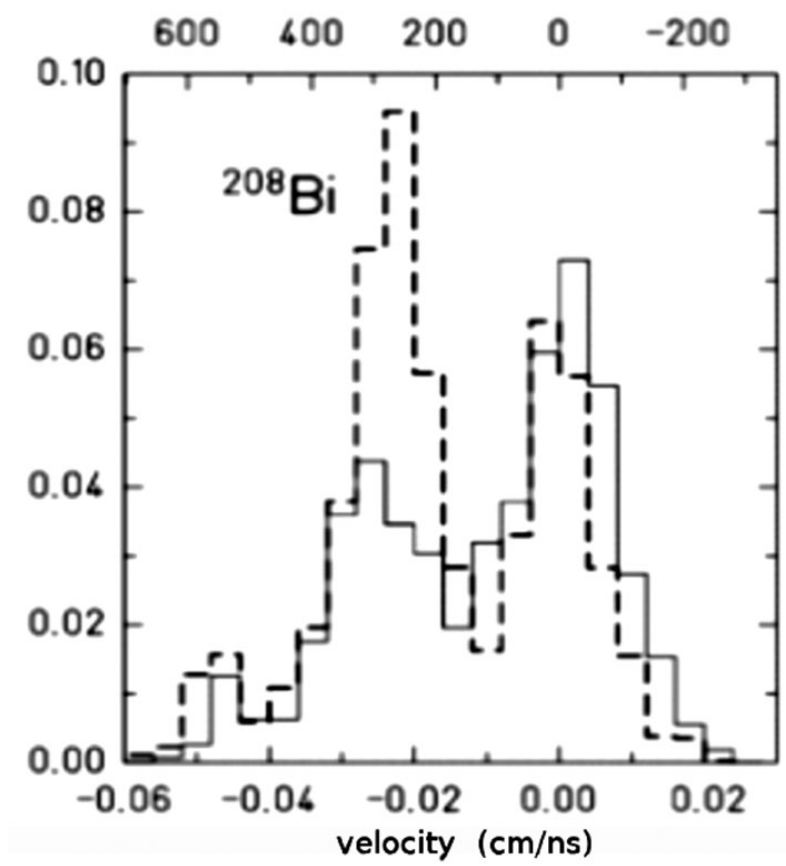

Fig. 13. Distribution of the longitudinal velocity of ${ }^{208} \mathrm{Bi}$ residues (with respect to the incident ${ }^{208} \mathrm{~Pb}$ velocity) produced in interactions of $1 \mathrm{GeV}$ per nucleon ${ }^{208} \mathrm{~Pb}$ nuclei with protons (full lines) and deuterons (dotted lines) targets. The distribution is expressed in arbitrary units and the velocities are given in $\mathrm{cm} / \mathrm{ns}$. The $\beta=v / c$ ratio is approximately equal to $v[\mathrm{~cm} / \mathrm{ns}] / 30$. The upper horizontal scale gives the relationship between $\Delta m$ and the recoil velocity (lower horizontal scale) as expressed by eq. (8). See text for details. Data from ref. [44].

The exact relation is not very transparent. In the case of $p+{ }^{208} \mathrm{~Pb} \rightarrow n+{ }^{208} \mathrm{Bi}$ reaction, one can make reasonable approximations $\left(E^{*} \ll M_{R}\right.$ and $\left.p_{R} \ll p_{0}\right)$, leading to:

$$
E^{*} \approx Q+\frac{p_{0} p_{R}}{\sqrt{p_{0}^{2}+m^{2}}} \approx Q+M \beta_{R} \frac{p_{0}}{\sqrt{p_{0}^{2}+m^{2}}},
$$

where $\beta_{R}$ is the velocity of the residue. We have used $p_{R} \ll M_{R}$ and $M \approx M_{R}$.

The peak in the velocity distribution around a vanishing recoil velocity can tentatively be interpreted on this way. The excitation energy should be small, less than the neutron emission threshold energy. Otherwise, ${ }^{208}$ Bi isotopes would not be observed. Therefore, the corresponding recoil velocity should be small.. For the particular case of the $p+{ }^{208} \mathrm{~Pb} \rightarrow n+{ }^{208} \mathrm{Bi}$ reaction, the recoil velocity cannot be smaller than $\sim 2 \times 10^{-5}$, due to a negative $Q$-value $\left(-3.66 \mathrm{MeV}\right.$, actually). On the other hand, the excitation energy of the ${ }^{208} \mathrm{Bi}$ residue should not be larger than the neutron separation energy, roughly $6.88 \mathrm{MeV}$. As a result, the recoil velocity $\beta_{R}$ (in direct kinematics) should be smaller than $\sim 6 \times 10^{-5}$ (or $v_{R} \leq \sim 0.0018 \mathrm{~cm} / \mathrm{ns}$ ). Note however that the experimental peak of the recoil velocity distribution in fig. 13 is much broader than indicated by these considerations, presumably because of a poor experimental resolution.

The second peak (on the left side of fig. 13) seems to correspond to an inelastic collision. The possible mechanisms have been discussed in sect. 3.3.2. A careful analysis reveals that the most probable mechanisms leading to a weakly excited ${ }^{208} \mathrm{Bi}$ residue are the $p n \rightarrow p \Delta^{0}, \Delta^{0} \rightarrow p \pi^{-}$and the similar $p n \rightarrow p \Delta^{0}, \Delta^{0} \rightarrow n \pi^{0}$ mechanism, subject of course to the kinematical constraints explained in this section ${ }^{7}$. In both cases, the pion should be emitted with its nucleon partner in the $\Delta^{0}$. Therefore, one may consider that a correlated pion-nucleon is emitted and that at the same time the other nucleon gets stuck in the residue, gifted with a small excitation energy. Let us consider the extreme case of vanishing excitation energy of the residue. For kinematical purposes, we may consider that the correlated pion-nucleon pair is equivalent to a $\Delta$ particle (with a definite mass $m^{*}$ ) since the sum of the pion and nucleon 4-momenta is equal to that of the parent particle. Let us assume that the residue is emitted in the forward direction. This is thus also the case for the correlated pair or " $\Delta$ " particle. Equation (4) for momentum conservation remains the same, whereas eq. (5) for energy conservation becomes

$$
\sqrt{p_{0}^{2}+m^{2}}+M=\sqrt{p_{R}^{2}+M_{R}^{2}}+\sqrt{p_{1}^{2}+m^{* 2}} .
$$

\footnotetext{
${ }^{7}$ The $p n \rightarrow n \Delta^{+}, \Delta^{+} \rightarrow p \pi^{0}$ mechanism, mentioned in sect. 3.3.2 is also possible, but seems less probable as the emitted nucleon and pion do not originate from the same $\Delta^{+}$resonance.
} 
Now writing $M_{R}=M-Q, m^{*}=m+\Delta m$ and assuming, as it is reasonable in our example, that $p_{R} \ll p_{0}$ and $Q \ll M$, one gets instead of eq. (6),

$$
\Delta m \approx \frac{p_{0} p_{R}}{m} \approx M \beta_{R} \frac{p_{0}}{m} .
$$

Basically, the excitation of the target or of the projectile gives the same linear variation with the residue velocity, except for a factor $m / \sqrt{p_{0}^{2}+m^{2}}$. Coming to numerical values, a value of $\Delta m \approx 330 \mathrm{MeV}$, roughly equal to the energy necessary to excite a nucleon to a $\Delta$ resonance, corresponds to a recoil velocity of $0.03 \mathrm{~cm} / \mathrm{ns}$, very close to the location of the left peak observed in fig. 13. All these considerations show that, in the $p+{ }^{208} \mathrm{~Pb}$ reacting system, an inelastic collision with suitable kinematics can lead to the emission of the "Delta" particle leaving a ${ }^{208} \mathrm{Bi}$ remnant with very small excitation energy. Therefore the excitation energy quoted at the top of fig. 13 does not correspond to the residue, but rather, in some sense, to the projectile.

Note, that in this case of inelastic scattering, the width of the peak in the velocity distribution seems to reflect the dispersion of the mass of the $\Delta$ resonance and dominates the width due to the possible limitation of the residue excitation energy below the neutron emission threshold energy.

Actually, the velocity distribution displayed in fig. 13 is the differential (with respect to the longitudinal velocity) cross section $\mathrm{d} \sigma / \mathrm{d} v / /$ for the production of ${ }^{208} \mathrm{Bi}\left({ }^{Z+1} A\right.$ residue in general). Summing over the longitudinal velocity and emission angles should yield the exclusive total ${ }^{Z+1} A$ production cross section. This check is not possible in the example above since the absolute normalisation of the distribution is not possible.

The two peaks in the velocity distributions of ${ }^{Z+1} A$ residues are related to the two peaks in neutron double differential cross sections at small angles, as illustrated in fig. 2. The quasi-elastic peak in the neutron spectra and the right hand side peak in fig. 13 are due the a single scattering collision. Actually, this is strictly true for the velocity spectra. For the neutron spectra, the quasi-elastic peak collects all neutrons which are emitted in the first collision, irrespective of what happens afterwards: several collisions may occur and other residues than the ${ }^{Z+1} A$ residues can be produced. It should be added that, in cascade models, most of the neutrons emitted in the quasi-elastic peak (more than 90 percent) are produced in single collision events. This has been verified in refs. $[16,18]$. Nevertheless, although all events contributing to the right hand side peak in the velocity distribution are among those events contributing to the elastic peak of the neutron double differential cross section, the former are less numerous, since quasi-elastic scattering refers to the forward emitted nucleon and does not require anything on the residue. To the best of our knowledge, this question has never been investigated. Similar considerations can be made for the quasi-inelastic peak in neutron spectra (fig. 2) and the left-hand side peak in fig. 13, although the dominance of single collision events is less pronounced, except of course for residues issued from fission.

The presence of two well-defined peaks in the longitudinal velocity distribution should also occur for ${ }^{Z} A$ residues, i.e. ${ }^{208} \mathrm{~Pb}$ residues in the example of the $p+{ }^{208} \mathrm{~Pb}$ system considered above. This is, of course, not possible to measure in reverse kinematics since the residue and the projectile are indistinguishable. Following the discussion above, a similar two-peak structure is also expected in the velocity distribution of ${ }^{Z-1}(A-1)$ and ${ }^{Z}(A-1)$ residues, as these isotopes can be made in single-scattering mechanisms (in the example above, this refers to ${ }^{207} \mathrm{Tl}$ and ${ }^{207} \mathrm{~Pb}$ isotopes). To the best of our knowledge these cases have not been studied in the same detail as for the Bi isotopes in ref. [44]. For all other residues, either the number of collisions is larger than one and/or evaporation is important. There are mutliple ways to generate a given isotope and the velocity distribution tends to have a Gaussian single-peaked shape, as this has been established by the GSI reverse kinematics measurements.

\section{Simple considerations about cross sections for single-scattering mechanisms}

Summarizing the discussion of sect. 3.3.6, only the following isotopes can be formed through single collision mechanisms: i) three $A+1$ isotopes, ${ }^{Z+2}(A+1),{ }^{Z+1}(A+1)$ and ${ }^{Z}(A+1)$, corresponding to the three yellow boxes of fig. 12 , ii) three isobars of the target nucleus ${ }^{Z+1} A,{ }^{Z} A$ and ${ }^{Z-1} A$, iii) two $A-1$ isobars, namely ${ }^{Z-1}(A-1)$ and ${ }^{Z}(A-1)$, corresponding to the one-nucleon removal cases.

As we explained, the production cross sections for these isotopes are rather satisfactorily described by INCL models (except of course for the ${ }^{Z} A$ case), especially in view of the spreading of the numerical values. Indeed $A+1$ isotopes are produced with cross sections of the order of a few tens of $\mu \mathrm{b}, A$ isotopes, with cross sections of the order of the mb and $A-1$ isotopes with cross sections of the order of few tens of mb. The values of the cross sections are stretching on almost five orders of magnitude. These trends could be related to the constraints on the single collision leading to these several isotopes. For $A-1$ isotopes, the colliding partners should basically avoid further collision. For $A$ isotopes, one of the colliding partners should avoid further collision, whereas the other should be stuck to the nucleus. Finally, for $A+1$ isotopes, both nucleons should be stuck to the nucleus.

Let us call $\sigma_{R}$ the total reaction cross section. In a model dominated by collisions, this is also the cross section for having at least one collision. Let us call $\sigma_{1}$ the cross section for having one collision and only one collision. This cross 
section is equal to the sum of the production cross section for all the isotopes mentioned above. We split this cross section as follow, with obvious notation,

$$
\sigma_{1}=\sigma_{A-1}+\sigma_{A}+\sigma_{A+1} .
$$

Since we are looking for general considerations, we do not distinguish the various isotopes. So the quantities in eq. (9) can be viewed as sums over isobar production cross sections, but relations involving specific isotopes may be written as well.

We concentrate now on the production of $A-1$ isotopes, i.e. the events corresponding to a quasi-free NN scattering. For the sake of our discussion, which aims to work out simple relations between cross sections, we do not distinguish neutrons and protons (as far as mechanisms are concerned). We can thus write

$$
\sigma_{A-1}=\sigma_{R} P_{n i}
$$

where $P_{n i}$ is simply the average probability of no further interaction for the particles issued from the first collision. The probability $P_{n i, 1}$ for the incident particle not interacting further can be calculated in a naive Glauber model. We just give here the value for the typical nuclear case (for which the product $x=\sigma_{N N} \rho_{0} R$ of the $N N$ total cross section, of the average nucleon density and of the nuclear radius satisfies $x \gg 1$ ). It writes [61]

$$
P_{n i 1} \approx \frac{1}{\left(\sigma_{N N} \rho_{0} R\right)^{2}}
$$

A similar formula can be found for $P_{n i 2}$, which is the probability for the target nucleon to avoid collisions after having been struck, in the same limit, $x \gg 1$ :

$$
P_{n i 2} \approx \frac{3}{2 \sigma_{N N} \rho_{0} R}
$$

Putting typical values yields $P_{n i, 1} \approx 0.06$. This estimate is not realistic, because the expression above is based on a sharp surface and neglects Pauli blocking. Actually, a proper treatment of the nuclear surface is crucial here, since the probability of no further interaction sensitively depends upon the impact parameter in the surface. We do not enter into the details here and simply concentrate on average quantities. Taking more realistic evaluations gives $P_{n i, 1} \approx 0.1$ and $P_{n i, 2} \approx 0.3$. The quantity $P_{n i}$ refers to the non-interaction of 2 or 3 particles. Thus typically, one expects $P_{n i} \approx 0.03-0.01$. Applying this simple rule to the $(p, 2 p)$ or $(p, p n)$ production gives values around 25 and $35 \mathrm{mb}$, respectively, which is indeed not far from the typical values encountered in sect. $3.3 .1^{8}$.

We turn now to the production of $A$ isotopes. In contrast to the case of the production of $A-1$ isotopes, instead of escaping the nucleus and further interaction, a nucleon should now get stuck in the target remnant. It should be stressed that the nucleon remains stuck not because it is making further collision and losing its energy, but because it is formed with a very small energy which does not allow it to escape. This requires a very stringent kinematical condition, which will not be fulfilled easily, especially at high incident energy. If we call $P_{s t}$ the probability for the fulfillment of this condition, we may write

$$
\sigma_{A}=\sigma_{A-1} \frac{P_{s t}}{P_{n i, 1}}
$$

There is no easy way to calculate or even estimate $P_{s t}$ (except with help of INC models, but not in the form of approximate transparent formulae). It should be small anyway, as it could be controled by a quantity like $\left(p_{F} / p_{0}\right)^{3}$. Taking typical values, this quantity is less than $10^{-2}$. If we look to the experimental values of $\sigma_{A-1}$ and $\sigma_{A}$, one finds $P_{s t} / P_{n i, 1} \approx 10^{-2}$, indicating that $P_{s t} \approx 10^{-3}$.

The same kind of considerations allows to write the following approximate reation:

$$
\sigma_{A+1}=\frac{1}{2} \sigma_{A} \frac{P_{s t}}{P_{n i, 1}}
$$

where the factor $1 / 2$ roughly accounts for the fact that only inelastic processes contribute to the production of $A+1$ isotopes. This relation is plainly consistent with the experimental values quoted above, if $P_{s t} \sim 10^{-3}$.

Let us finally notice that since $\sigma_{A-1} \gg \sigma_{A} \gg \sigma_{A+1}$, combining eqs. (9) and (10) allows to write:

$$
\sigma_{1} \cong \sigma_{R} P_{n i} \cong \sigma_{A-1}
$$

\footnotetext{
${ }^{8}$ Equation (10) is aimed at giving order of magnitude relationship. It does not distinguish, for instance, between neutrons and protons. Actually, if one corrects for this, one has to introduce, in first approximation a factor $Z / A$ or $N / A$ in the rhs of eq. (10) for $(p, 2 p)$ or $(p, p n)$, respectively. These factors have been introduced in our numerical considerations.
} 


\section{Single scattering approximation: A way to discuss missing features in INC models}

As we have seen, INCL is rather successful in describing many observables, even when the available phase space is reduced. For the residue production cross sections, this is particularly transparent, as underlined in the previous section. It is therefore annoying and surprising that the model fails on observables which are dominated by single scattering mechanisms. In this section we try to identify what is missing in INCL and to present possible remedies. For that purpose, it is interesting to start with a single scattering approximation of multiple scattering theory, which presents the advantage of being formulated at the quantum level. To be more specific, we will deal with inclusive nucleon quasi-elastic scattering, similar considerations being possible for the other observables dominated by single scattering.

There are several versions of the multiple scattering theory, among which the most utilized ones are those of refs. [62-65]. The single scattering approximation assumes basically the same form in all versions for the differential cross section of the inclusive $N+{ }^{Z} A \rightarrow N+X$ scattering:

$$
\frac{\mathrm{d} \sigma}{\mathrm{d}^{3} k_{1}}=\frac{(2 \pi)^{4}}{\hbar v_{0}} \sum_{n} \delta\left(E_{1}-E_{1}^{0}+E_{n 0}\right)\left|\left\langle\boldsymbol{k}_{1} n|T| \boldsymbol{k}_{1}^{\mathbf{0}} 0\right\rangle\right|^{2},
$$

where $\boldsymbol{k}_{\mathbf{1}}^{\mathbf{0}}$ denotes both the momentum of the incident nucleon and the quantum state of this particle (here a plane wave), $\boldsymbol{k}_{\mathbf{1}}$ plays the same role for the detected nucleon, $|0\rangle$ is the target ground state, $|n\rangle$ stands in principle for any excited state, $E_{1}^{0}$ is the energy of the incident nucleon, $E_{1}$ is the energy of the detected nucleon, $E_{n 0}=E_{n}-E_{0}$ is the excitation energy of the state $|n\rangle^{9}$ and $v_{0}$ is the relative velocity in the incident channel. The operator $T$ is defined by

$$
T=\sum_{j=1}^{A} t_{p j},
$$

where $t_{p j}$ is the $T$-operator for the elastic scattering of the incident particle $p$ with the $j$-th nucleon of the target in the nucleus medium.

From now on, we will write approximate relations which, sometimes, will not be rigorous. Our aim is to illustrate what physical features of the quasi-elastic scattering are retained in INCL and which ones are neglected. To do so, eq. (16) is not really appropriate. Actually, this equation is valid in the weak coupling approximation. It assumes that a nucleon is emitted in the single collision under consideration. It neglects distortion of the incident proton wave prior to the collision and the distortion of the outgoing nucleon after the collision. This is reflected by the use of plane waves $\boldsymbol{k}_{1}$ and $\boldsymbol{k}_{1}^{\mathbf{0}}$ in expression (16). Taking care of these possible distortions means that the incoming nucleon has a smaller probability to make a single collision emitting directly a nucleon and that the outgoing nucleon has less chance to escape. This effect can be taken into account by introducing distorted waves instead of plane waves [66]. These waves are supposed to be solutions of the optical model and correspond to a decreasing flux as the waves propagate. In order to keep the formulation as transparent as possible, we will introduce more simply a probability $P_{\text {esc }}$ of escaping collisions before and after the single "useful" collision, as in the preceding section. We will even consider that this probability is the same for all kinematical conditions or rather that $P_{\text {esc }}$ represents an average.

Further discussions are based on the free Fermi gas picture of the nucleus, which is also the basic picture used in INC models. We refer to ref. [67] for details. Target excited sates $|n\rangle$ are particle-hole states. The operator $T$ in eq. (17) can be considered as a one-body operator in the target Fock space. Therefore, only one particle-one hole states can be connected to the ground state in the matrix element of $T$ in eq. (16). Furthermore conservation of momentum and Pauli principle imply that the states $|n\rangle$ are restricted to

$$
|n\rangle=a_{\boldsymbol{k}-\boldsymbol{q}}^{+} a_{\boldsymbol{k}}|0\rangle,
$$

with $k<k_{F}$ and $|\boldsymbol{k}-\boldsymbol{q}|>k_{F}$. In these relations, $k_{F}$ is the Fermi momentum and $\boldsymbol{q}=\boldsymbol{k}_{\mathbf{1}}-\boldsymbol{k}_{\mathbf{1}}^{\mathbf{0}}$ is the momentum transferred to the incoming nucleon. One can then write, for a given transferred momentum $\boldsymbol{q}$ :

$$
\begin{aligned}
& \sum_{n} \delta\left(E_{1}-E_{1}^{0}-E_{n 0}\right)\left|\left\langle\boldsymbol{k}_{1} n|T| \boldsymbol{k}_{1}^{\mathbf{0}} 0\right\rangle\right|^{2} \\
& =\sum_{\boldsymbol{k}} \delta\left(E_{1}-E_{1}^{0}+\varepsilon_{|\boldsymbol{k}-\boldsymbol{q}|}-\varepsilon_{k}\right)\left|\left\langle\boldsymbol{k}_{\mathbf{1}} \boldsymbol{k}-\boldsymbol{q}\left|t_{p}\right| \boldsymbol{k}_{\mathbf{1}}^{\mathbf{0}} \boldsymbol{k}\right\rangle\right|^{2} n_{\boldsymbol{k}}^{2}\left(1-n_{\boldsymbol{k}-\boldsymbol{q}}\right)^{2} \\
& =\sum_{\boldsymbol{k}} \delta\left(E_{1}-E_{1}^{0}+\varepsilon_{|\boldsymbol{k}-\boldsymbol{q}|}-\varepsilon_{k}\right)\left|\left\langle\boldsymbol{k}_{\mathbf{1}}^{\mathbf{0}}+\boldsymbol{q} \boldsymbol{k}-\boldsymbol{q}\left|t_{p}(\tilde{E})\right| \boldsymbol{k}_{\mathbf{1}}^{\mathbf{0}} \boldsymbol{k}\right\rangle\right|^{2} n_{\boldsymbol{k}}^{2}\left(1-n_{\boldsymbol{k}-\boldsymbol{q}}\right)^{2} .
\end{aligned}
$$

\footnotetext{
${ }^{9}$ We have neglected the recoil energy here.
} 
In this equation, $\varepsilon_{\boldsymbol{k}}$ is the single-particle energy of the target nucleon with momentum $\boldsymbol{k}$ and $n_{\boldsymbol{k}}=\left\langle 0\left|a_{\boldsymbol{k}}^{+} a_{\boldsymbol{k}}\right| 0\right\rangle$. In the free Fermi gas model, $n_{\boldsymbol{k}}$ reduces to

$$
n_{\boldsymbol{k}}=\theta_{H}\left(k_{F}-k\right),
$$

where $\theta_{H}$ is the Heaviside function, and satisfies $n_{\boldsymbol{k}}^{2}=n_{\boldsymbol{k}}$ and $\left(1-n_{\boldsymbol{k}}\right)^{2}=1-n_{\boldsymbol{k}}$. Using eqs. (16) and (19), one gets

$$
\frac{\mathrm{d} \sigma}{\mathrm{d}^{3} k_{1}}=\frac{(2 \pi)^{4}}{\hbar v_{0}} P_{\mathrm{esc}} \sum_{\boldsymbol{k}} \delta\left(E_{1}-E_{1}^{0}+\varepsilon_{|\boldsymbol{k}-\boldsymbol{q}|}-\varepsilon_{k}\right)\left|\left\langle\boldsymbol{k}_{\mathbf{1}}^{\mathbf{0}}+\boldsymbol{q} \boldsymbol{k}-\boldsymbol{q}\left|t_{p}(\tilde{E})\right| \boldsymbol{k}_{\mathbf{1}}^{\mathbf{0}} \boldsymbol{k}\right\rangle\right|^{2} n_{\boldsymbol{k}}^{2}\left(1-n_{\boldsymbol{k}-\boldsymbol{q}}\right)^{2} .
$$

The $t$-matrix element is off-shell. The energy $\tilde{E}$ is basically the incident energy plus the average single-particle energy and does not correspond to the energy of free colliding nucleons with the indicated momenta. Usually, it is assumed that the matrix element does not depend very much on the kinematical off-shell variable and the matrix element is put on-shell, with $\tilde{E}=E_{O N}$, the on-shell energy. This is what is done in INCL4. We will come back to this point.

In principle, the states $|\boldsymbol{k}\rangle$ and $|\boldsymbol{k}-\boldsymbol{q}\rangle$ are single-particle states confined inside the target volume. The connection of the $t$-matrix elements with free nucleon-nucleon cross sections requires to use plane waves. The passage from one to the other involves the introduction of the nuclear density and complicates the formulae. The final result we want to derive can be obtained by taking the continuous limit of the free Fermi gas confined to a volume $\mathrm{V}$. It is well known that the sum over states $|\boldsymbol{k}\rangle$ (below Fermi level) is replaced by an integration over $\boldsymbol{k}$ multiplied by a factor $V /(2 \pi)^{3}$. One does get, from eq. (21),

$$
\frac{\mathrm{d} \sigma}{\mathrm{d}^{3} k_{1}}=\frac{(2 \pi)^{4}}{\hbar v_{0}} P_{\mathrm{esc}} \frac{V}{(2 \pi)^{3}} \int_{k<k_{F}} \delta\left(E_{1}-E_{1}^{0}+\varepsilon_{|\boldsymbol{k}-\boldsymbol{q}|}-\varepsilon_{k}\right)\left|\left\langle\boldsymbol{k}_{\mathbf{1}}^{\mathbf{0}}+\boldsymbol{q} \boldsymbol{k}-\boldsymbol{q}\left|t_{p}(\tilde{E})\right| \boldsymbol{k}_{\mathbf{1}}^{\mathbf{0}} \boldsymbol{k}\right\rangle\right|^{2} n_{\boldsymbol{k}}^{2}\left(1-n_{\boldsymbol{k}-\boldsymbol{q}}\right)^{2} .
$$

Finally, we will use the reduced variable $x=k / k_{F}$ and the free Fermi gas model implying $n_{x}=n_{x}^{2}$ and

$$
A \approx \frac{V k_{F}^{3}}{3 \pi^{2}}
$$

The on-shell $T$-matrix can be related to the free NN cross section (with the first factor in eq. (22)). One finally gets

$$
\frac{\mathrm{d} \sigma}{\mathrm{d}^{3} k_{1}} \approx P_{\mathrm{esc}} A \frac{\mathrm{d} \sigma_{O N}^{N N}}{\mathrm{~d}^{3} k_{1}} S(q, \omega)
$$

where

$$
S(q, \omega)=\int_{x<1} \delta\left(\omega-\varepsilon_{|\boldsymbol{k}-\boldsymbol{q}|}+\varepsilon_{k}\right) n_{\boldsymbol{k}}\left(1-n_{\boldsymbol{k}-\boldsymbol{q}}\right)
$$

is the so-called Lindhard function, where $\omega=E_{1}^{0}-E_{1}$ and where $\sigma_{O N}^{N N}$ is the free on-shell nucleon-nucleon cross section.

Quite often, this formula is written by indroducing a number $A_{\text {eff }}$ of "effective target nucleons", i.e. the nucleons which are apparently "active" in this approximation,

$$
A_{\text {eff }}=P_{\text {esc }} A \text {. }
$$

Equation (24) is very simple and has the advantage of presenting the basic features of single scattering processes. It nevertheless has an obvious weakness: it relies on other models for predicting the number of effective nucleons. Usually, this is done using simple models, such as the Glauber model. Since the evaluation of this quantity involves geometrical properties of nuclei and nucleon-nucleon total cross sections, it should be emphasized that INC models are probably the best predictive models for this quantity.

Nevertheless, expression (24) can be helpful for discussing the possible weak points of INCL4. The latter may primarily deal with the individual cross section controling the single collision and with the available phase space.

According to the above discussion, one should in principle use a off-shell cross section (or rather an off-shell $t$ matrix). The most obvious consequence would be to use an energy variable which is different from the on-shell energy (the energy calculated from the momenta as in free space kinematics). The off-shell energy is smaller than the on-shell energy, basically because the target nucleon is moving inside the attractive nuclear mean field. The difference is the depth of the nuclear mean field, which is around 40 to $50 \mathrm{MeV}^{10}$. It is not clear whether such an effect is important. The off-shell extension of the $t$-matrix has not been studied in detail and through to the use of some nucleon-nucleon potentials only. The answer is thus not clear as it may be potential-dependent. It seems that, on the average, the effect is not very important [68]. This effect is not introduced in INCL4, although a similar correction is applied on

\footnotetext{
10 High-energy incident nucleons experience a mean field which is basically vanishing.
} 
the first collision. In the jargon of the experts, it is called the LocE recipe [19,24]. It basically consists in performing an on-shell collision after having decreased the momenta of the colliding nucleons in such a way that the energy of the colliding pair is roughly equal to the off-shell energy discussed above. A somehow symmetric correction is applied after collision in order to restore momentum conservation. The locE recipe has been introduced for somehow correcting the potential energy of the nucleons in the nuclear surface, which is not totally satisfactory in INCL4 (see below). Actually this recipe has the effect of enhancing the probability of collisions in the nuclear surface at low energy, helping so to have a correct total reaction cross section. This was the main incentive to introduce the LocE recipe. Unfortunately, it turned out that this recipe has a rather bad influence on the description of the quasi-elastic peak, as can be seen from fig. 3. The main effect is a reduction of the width of the peak, by almost a factor 2. There is also an apparent shift of the peak toward higher neutron energy. However, the experimental position of the peak may be shifted to lower energy because of the thickness of the target used in ref. [36]. The latter might be responsible for downard shift of 20 to $40 \mathrm{MeV}[19,69]$, but this has never been evaluated in detail.

Concerning the nucleon-nucleon cross section to be used, one cannot rule out the often advocated correction for medium effects. On the theoretical side, only medium effects embodied by the so-called Brueckner G-matrix, i.e. those which are linked with the Pauli blocking in intermediate states in the two-body scattering process, have been studied [73-77]. They have a sizeable effect at low energy, but as expected, the effect is vanishing rapidly as the energy increases. Other phenomenological studies have been done, basically by looking at effects under an artificial variation of the value of the NN cross sections. Under reasonable variations, particle spectra are only mildly affected and in some limited region of phase space [78]. To the best of our knowledge, there is no study of the effects on residue production.

The other aspect of eq. (24), related to the last factor $S(q, \omega)$, deals with the space of target states under consideration. This formula is obtained by using pure free Fermi gas model, in the limit of infinite nuclear matter to be more precise. The facteur $S(q, \omega)$ also takes account of the Pauli principle in the $1 p$ - $1 h$ excitations of the target. Rougly speaking, the function $S(q, \omega)$ presents maxima around $\omega=q^{2} / 2 m$ and is significantly different from zero in an interval, which roughly corresponds to the width of the Fermi sea [70].

Although INCL4 relies on the free Fermi gas model, it does not correspond exactly to expression (24), but rather to

$$
\frac{\mathrm{d} \sigma}{\mathrm{d}^{3} k_{1}} \approx \frac{\mathrm{d} \sigma_{O N}^{N N}}{\mathrm{~d}^{3} k_{1}} \int \mathrm{d}^{3} \boldsymbol{r} \rho(\boldsymbol{r}) P_{\mathrm{esc}}(\boldsymbol{r}) S_{r p}(q, \omega),
$$

with

$$
S_{r p}(q, \omega)=\int_{x<1} \mathrm{~d}^{3} \boldsymbol{k} \delta\left(\omega-\varepsilon_{|\boldsymbol{k}-\boldsymbol{q}|}+\varepsilon_{k}\right) f(\boldsymbol{r}, \boldsymbol{k})(1-f(\boldsymbol{r}, \boldsymbol{k})) .
$$

In the last equation, $f(\boldsymbol{r}, \boldsymbol{k})$ is the distribution function of the nucleons in phase space, normalized as $\int \mathrm{d}^{3} \boldsymbol{r} \mathrm{d}^{3} \boldsymbol{k} /(2 \pi)^{3} f(\boldsymbol{r}, \boldsymbol{k})=A$. We do not have a rigorous derivation of eq. (27), but we have plausibility arguments. First, collisions occur at a definite location in INCL4 and therefore the probability of having a single collision at point $\boldsymbol{r}$ depends on $\boldsymbol{r}$. Second, as explained in sect. 2.2, the Pauli blocking factors in INCL4 are calculated using phase space density. Third, formula (27) reduces to expression $(24)$, when $f(\boldsymbol{r}, \boldsymbol{k}) \rightarrow \theta_{H}\left(k_{F}-k\right) \theta_{H}(R-r)$, the typical phase space distribution of (infinite) uniform Fermi gas, provided $P_{\text {esc }}$ in eq. (24) is considered as the average value of $P_{\text {esc }}(\boldsymbol{r})$ in eq. (27).

We now want to discuss the strong and weak points of INCL4 in comparison with eqs. (27) and (24), trying first to make our comments as general as possible.

A very detailed analysis [70] of the predictions of expression (24), using numerical values of $A_{\text {eff }}$ provided by INCL4.2 [19], reveals that this formula gives rather good results for quasi-elastic $(p, n)$ double differential cross sections at not too small angles, i.e. for $\theta \gtrsim 10^{\circ}$, but fails miserably at zero degree. The same situation roughly holds for INCL4.6 (or, more or less equivalently for expression (27)), when the LocE recipe is avoided, as can be seen from fig. 3. The effect of LocE is perhaps easy to understand from our discussion above, since it effectively diminishes the Fermi motion of the target nucleons in the nuclear surface.

Nevertheless, with or without LocE, it seems that the quasi-elastic peak at very small angles is not reproduced by INCL4 and alike models, essentially based on collisions and free Fermi gas. This issue has received some attention in the past [79-83]. In these works, it is advocated that the free Fermi gas model is not appropriate to describe the quasi-elastic peak and that the latter would rather arise from surface collective excitations mixed with the spin dependence of nucleon-nucleon cross section. Although this explanation is somewhat strange (collective effects seem at first sight hardly excited by high-energy particles), it nevertheless improved the description, even if perfect agreement was not obtained. In any case, it would be desirable to go beyond the free Fermi gas picture. Indeed the latter is only a first approximation to the structure of the nuclei. The analysis of the $\left(e, e^{\prime} p\right)$ reactions on the magic nucleus ${ }^{208} \mathrm{~Pb}$ at NIKHEF [84] has indicated a depletion of $15 \%$ to $20 \%$ of the Fermi sea. In other words, it seems that the quantities $n_{k}$ of eq. (24) (or equivalently $\int \mathrm{d}^{3} \boldsymbol{r} f(\boldsymbol{r}, \boldsymbol{k})$ in eq. (27) are not equal to 0 or 1 . This is indicative of the importance of residual interactions between the nucleons, which are no longer on shell. In principle, there is a well-defined theoretical framework for handling off-shell nucleons, based on the Green function formalism [29,85,86, 30,31 , in which a quantity related to $f(\boldsymbol{r}, \boldsymbol{k})$ should be supplemented with the so-called strength function, describing 
the off-shell behavior, in order to have a complete description of nuclear dynamics. This results in complex coupled equations linking the two quantities. This formalism is too complex to be solved in realistic situations and there is no clear way the expected effects can be simulated in INC models. But it is clear that these effects are not negligible [86, 87]. This line of investigation should deserve interest in the next future.

We want to mention that, before entering this huge task, improving the INC models while still sticking to the free Fermi gas model may be worth to be studied. One issue is perhaps the best choice for the initial phase space distribution function $f(\boldsymbol{r}, \boldsymbol{k})$. Several choices are possible and consistent with the free gas picture. The popular quasiclassical distribution is

$$
f(\boldsymbol{r}, \boldsymbol{k})=C \theta_{H}\left(\mu-k^{2} / 2 m-V(r)\right),
$$

where $\mu$ is the chemical potential, $C$ is the normalization constant (such that $\int f=A$ ) and $V(r)$ is the average potential. In simulations, this distribution corresponds to particles with momenta taken randomly in a sphere of radius $k_{F}$ in k-space and moving classically and independently in the potential well $V(r)$. In INCL4, the following distribution is used:

$$
f(\boldsymbol{r}, \boldsymbol{k})=A \frac{\theta_{H}(R(k)-r) \theta_{H}\left(k_{F}-k\right)}{\left(\frac{4 \pi}{3}\right)^{2} R^{3}(k) k_{F}^{3}},
$$

where $R(k)$ is such that the integral $\int f(\boldsymbol{r}, \boldsymbol{k}) \mathrm{d}^{3} k$ is equal to a prescribed density $\rho(r)$. Without entering into details (given in ref. [19]), it is sufficient here to say that this distribution corresponds to simulations in which the momentum of each of the $\mathrm{A}$ nucleons is first taken at random in a sphere of radius $k_{F}$ and afterwards its position is taken at random in a sphere of radius $R(k)$. It is also shown in ref. [19] that the distribution (30) remains invariant in time, in the absence of collisions, if a nucleon of momentum $\mathrm{k}$ feels a square well of constant depth $V_{0}(k)$ and constant radius $R(k)$ (which may depend upon $k$ as indicated). In other words, a nucleon of momentum $k$ moves in its potential and feels a classical turning point on the sphere of radius $R(k)$. In ref. [26], it is noticed that this choice neglects a well known quantum effect, namely the possible location of a nucleon beyond the value of its classical turning point. In the same reference, it is proposed that the value of the "effective" turning point in eq. (30) be taken at random with a value which, on the average, is equal to $R(k)$. Without entering into details, which can be found in ref. [26], this roughly amounts to considering a distribution function of the form

$$
f(\boldsymbol{r}, \boldsymbol{k}, \xi)=A f_{N}(\xi, R(k), a) \frac{\theta_{H}(\xi-r) \theta_{H}\left(k_{F}-k\right)}{\left(\frac{4 \pi}{3}\right)^{2} R^{3}(k) k_{F}^{3}},
$$

where $\xi$ is an auxiliary random variable following a Gaussian distribution $f_{N}$, of mean $R(k)$ and rms $a$. The parameter $a$ and the possible mixing of the two distributions (30) and (31) are adjusted to fit the properties of the resulting distribution on those of quantum particles in a realistic Saxon-Woods potential. One can easily realize that a net effect is that it is possible to find a nucleon of a given energy further away from the center than previously, i.e. farther in the outskirts of the nuclear surface. Let us now discuss the consequences for one-nucleon removal reactions. As we explained above, these reactions may be viewed as resulting from a single nucleon-nucleon collision with no further interaction of any sort. Let us assume that this collision occurs at a distance $r$ from the center and that the target nucleon has an energy $\varepsilon_{k}$. In the original INCL4.6 model, corresponding to the initial distribution (30), the excitation energy left after this collision is simply the difference between the Fermi energy and $\varepsilon_{k}$, provided, of course, the location of the collision is within the turning point of the struck nucleon $R(k)$. In other words, the expected excitation energy is

$$
E^{*}=\left(\varepsilon_{F}-\varepsilon_{k}\right) \theta_{H}(R(k)-r)
$$

With the modified distribution this expected excitation energy becomes

$$
E^{*}=\left(\varepsilon_{F}-\varepsilon_{k}\right) \overline{\theta_{H}(\xi-r)},
$$

where the bar indicates averaging over the distribution $f_{N}$, for a fixed value of $k$. It is easy to see that the excitation energy may differ from zero in the second case, whereas it strictly vanishes when $r>R(k)$, in the previous case. In simple words, the fluctuations allow drilling (by a single collision) deeper holes in the surface. The excitation energy may thus be larger in the case of distribution (31) and the remnant has thus more chance to evaporate a neutron. In conclusion, fluctuations decrease the one-nucleon removal cross section. This is studied in detail in ref. [26]. It is shown indeed that the one-proton removal cross sections are really improved (but not suffciently) when calculated on this way. On the other hand, the one-neutron removal cross sections are less changed than for proton removal, but they were less off experimental values. This is an encouraging progress, but which still demands further investigation.

To conclude this section, we can say that we more or less arrive at the conclusion that INCL does not give a good description of the single scattering events presumably because some quantum effects are missing, essentially those linked with the off-shell behaviour of interacting nucleons. If this argument is right, it does not automatically explain why the other kinds of events (multiple scattering events) are well described, often in sharp contrast. One 
may anticipate on the possibility that quantum corrections on multiple scatterings are likely giving contributions with random signs leading to cancellations. This is expected, but by no means guaranteed, even if this argument is often presented as a justification of the validity of the INC models.

Before closing this section, it is perhaps the place to say a few words about evaporation models in the context of this paper. Presented results are obtained mainly by the INCL4.6 + ABLA07 combined model, as stated in the Introduction. We pay attention to the performances of the INCL4.6. One may wonder whether the choice of the ABLA07 model matters. Probably not. Indeed, the analysis of the single-scattering events reveals the importance of escaping or sticking probabilities, which are outputs of INCL. The evaporation model only enters for determining whether a low excitation remnant will decay or not. This is largely independent of the detail of evaporation models, because this essentially depends upon neutron emission threshold energies, which should basically be the same in all evaporation models. This statement may not be strictly true, since, for special nuclei, other decay modes, like alpha decay, may happen to be competitive with neutron emission, even at low excitation energy.

\section{A legacy of ANDES. The importance of single-scattering events in macroscopic applications}

We want here to discuss an unexpected result involving macroscopic spallation reactions, which singles out the importance of single scattering events. This result has been discovered in the frame of the ANDES research program. The purpose of the ANDES FP7-EURATOM project was "to address the nuclear data needs associated to the new reactors and new fuel cycles supported by SNETP, in its strategic research agenda and in the ESNII proposal". In the final summary report [88], it is said that "ANDES has combined a reduced group of selected differential measurements, the improvement in uncertainties and covariance's within the evaluation process and the validation of present and new data libraries using integral experiments, to improve most critical nuclear data to approach the level of accuracies required by the new reactors and system promoted by ESNII and the SNETP". In the frame of this program, a workpackage was devoted to the improvement of nuclear reaction models and, in particular of INCL. It was foreseen to benchmark the improvements of INCL4.6 by comparing with well-chosen thin and thick target data. To achieve the last task, the interest has been put on the ISOLDE IS419 experiment, which was devoted to the study of the production and release rates of volatile elements from a liquid $\mathrm{Pb}-\mathrm{Bi}$ eutectic target irradiated with proton beams of 1 and $1.4 \mathrm{GeV}$ [89-91].

We want here to discuss the results concerning the production of astatine isotopes in such targets. This topic was selected by the ANDES project because astatine poses serious radiotoxicity problems, due to its relatively high volatility, to the intermediate values of the lifetime of most of its isotopes and to its decay to Po isotopes.

In thin targets, At isotopes can be produced by ${ }^{209} \mathrm{Bi}\left(p, \pi^{-} x n\right)$ reactions. For $x=0$, leading to ${ }^{210} \mathrm{At}$, this is a special case of the production of ${ }^{Z+2} A$ isotopes that we discussed in sect. 3.3.3, corresponding to a single inelastic scattering $p n \rightarrow p \Delta^{0}$, followed by $\Delta^{0} \rightarrow p \pi^{-}$, with the conditions that the two nucleons remain stuck in the target with small energy, contributing to an excitation energy below neutron threshold. The $x>0$ cases correspond to either a similar scenario with larger excitation energy and/or to multiple scattering mechanisms with emission of nucleons. In order to have a good theoretical description of ${ }^{209} \mathrm{Bi}\left(p, \pi^{-} x n\right)$ reactions, it is absolutely necessary to have a reliable pion production mechanism. This is the case in INCL4.6, which is able to describe inclusive pion production [24], but also $\left(p, \pi^{-} x n\right)$ cross sections, which requires also a good description of the "sticking" of the colliding nucleon, as we just explained. Figure 10 illustrates this point. We have to stress again that the agreement between calculations and experiment, although not perfect, can be considered as a very good performance for a simulation model in view of the small size of the cross section and the delicate features of the production mechanism. Predictions of INCL4.6 concerning $\left(p, \pi^{-} x n\right)$ cross sections for other thin targets can be found in refs. [60,93,94].

In thick lead-bismuth eutectic (LBE) targets, there is another channel for the production of $A t$ isotopes, namely and mainly secondary ${ }^{209} \mathrm{Bi}(\alpha, x n)$ and ${ }^{209} \mathrm{Bi}\left({ }^{3} \mathrm{He}, x n\right)$ reactions induced by $\alpha$ and ${ }^{3} \mathrm{He}$ particles issued from primary collisions. A detailed analysis of the importance of the respective channels and of the various features of the respective mechanisms can be found in ref. [60]. We just want here to single out the salient features.

Release rates of astatine isotopes were measured in the Isolde experiment. However, the diffusion time of astatine in LBE is not known, which complicates comparison of calculations with experiment. This was tackled carefully in ref. [60]. A satisfactory agreement can be found when the diffusion time is assumed to be of the order of 10 hours, as illustrated by the magenta line in fig. 14. This prediction of the diffusion time of astatine in LBE is an unexpected result of the ANDES program. Of course, the determined value $(10 \mathrm{~h})$ should be checked by chemical measurements.

Figure 15 gives the contributions of the various channels to the astatine production, as predicted by INCL4.6ABLA07. It can be seen that the $\left(p, \pi^{-} x n\right)$ channel basically feed preferentially the low-mass isotopes whereas the $(\alpha, x n)$ and $\left({ }^{3} \mathrm{He}, x n\right)$ channels are predominantly feeding high-mass isotopes. It is one of the remarkable outputs of the ANDES program to have demonstrated the importance of secondary reactions and to have emphasized the necessity of having reliable models for the production and the interaction of lcp's. Calculations neglecting this point are unable to reproduce astatine data [95]. However, the work of ref. [60] and fig. 15 also show that a good reproduction of the data requires the introduction of the $\left(p, \pi^{-}\right)$channel and a reliable description of the corresponding single-scattering events. 


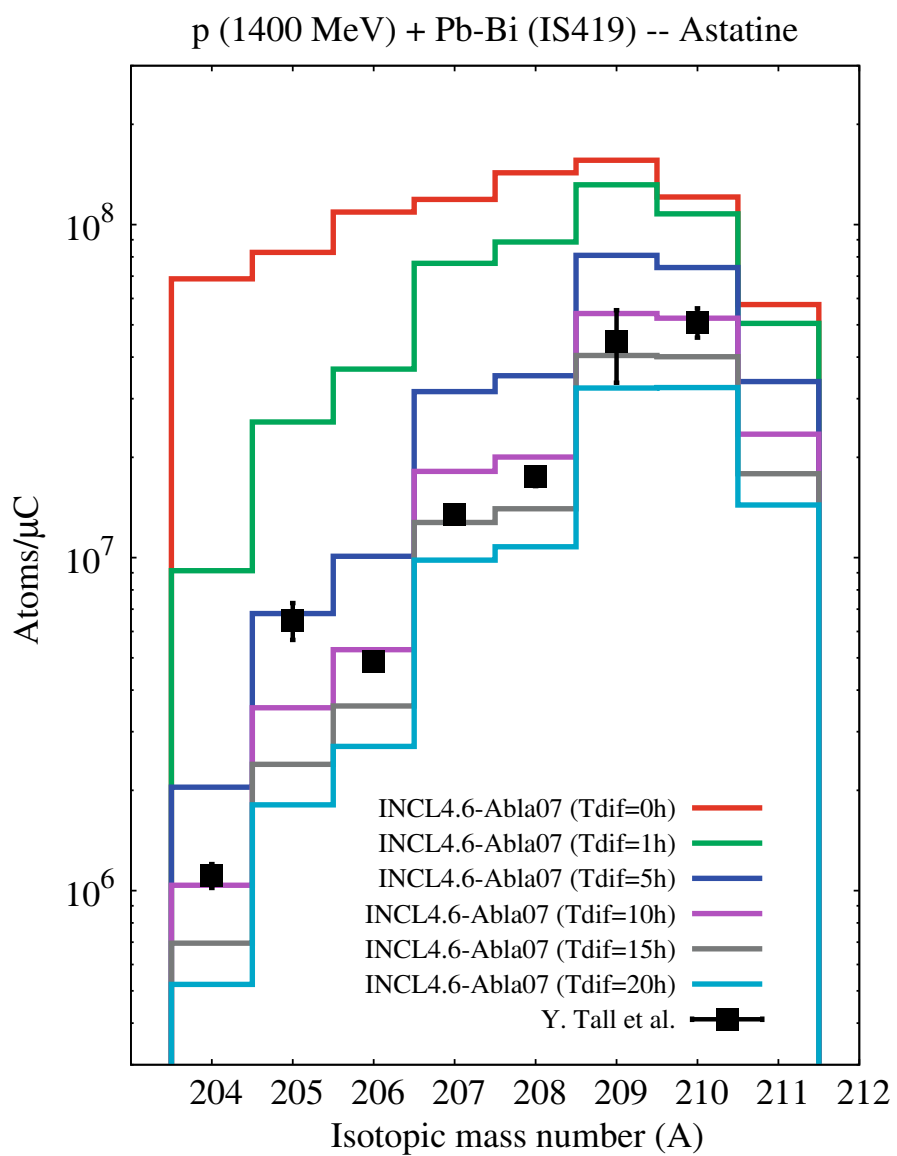

Fig. 14. Astatine release rates measured by the ISOLDE Collaboration [92] at $1.4 \mathrm{GeV}$ compared to MCNPX simulations, using INCL4.6-ABLA07. The solid red line is the yield given by the simulation assuming no diffusion and no decay. The other lines correspond to indicated values of the diffusion time and taking account of the decay of unstable isotopes. See [60] for detail.

\section{Conclusion}

This paper provides a review on an intriguing problem which attracted the attention of physicists regularly in the last fifteen years. The problem was probably raised for the first time in ref. [19], with some detail. It refers to the quasi-elastic peak that is observed in neutron spectra for proton-induced reactions. This peak, whose shape is grossly consistent with the nucleon-nucleon kinematics, is attributed to the first collision between the incident proton and a target neutron. The properties of the peak were analyzed in the single-scattering approximation with some success, as explained in sect. 5 above. This feature was considered as a strong indication of the dominance of the collision dynamics in the first stage of spallation reactions [96,97]. This dominance is supported by the increasing successes of the INC models. However, as underlined in ref. [19], the INCL4.2 model is able to reproduce quite well the gross features of the neutron spectra, but fails clearly in the predictions of the quasi-elastic peak. In particular, the attention is drawn on the paradox embodied in this observation: the quasi-elastic peak, generated by single scattering events, is less satisfactorily reproduced (by a pure collision model) than the rest of the neutron spectra, which likely results from multiple collision events.

This observation was followed by several similar ones, which showed that for other observables, the predictions of INC models are less good when they are linked with single scattering events ${ }^{11}$. The most spectacular example, which has been emphasized during the ANDES program, is the one proton removal cross sections, which are overpredicted by INC models, whereas the predictions for the production of neighbouring isotopes are clearly satisfactory. This is also a result of the ANDES program to have corroborated that the failures of the INCL model are generally occuring for observables which are connected with single collision events. The present paper essentially reports on the developments made during the ANDES program. It presents a review of the most important observables which are linked to single scattering events. In particular, the residues whose production cross sections are falling in this class of observables

\footnotetext{
11 Another interesting observable, somehow related to the quasi-elastic scattering is the quasi-free scattering, when two nucleon are detected simultenously with a quasi-free kinematics (see ref. [98], for an example) will be treated in a subsequent publication.
} 


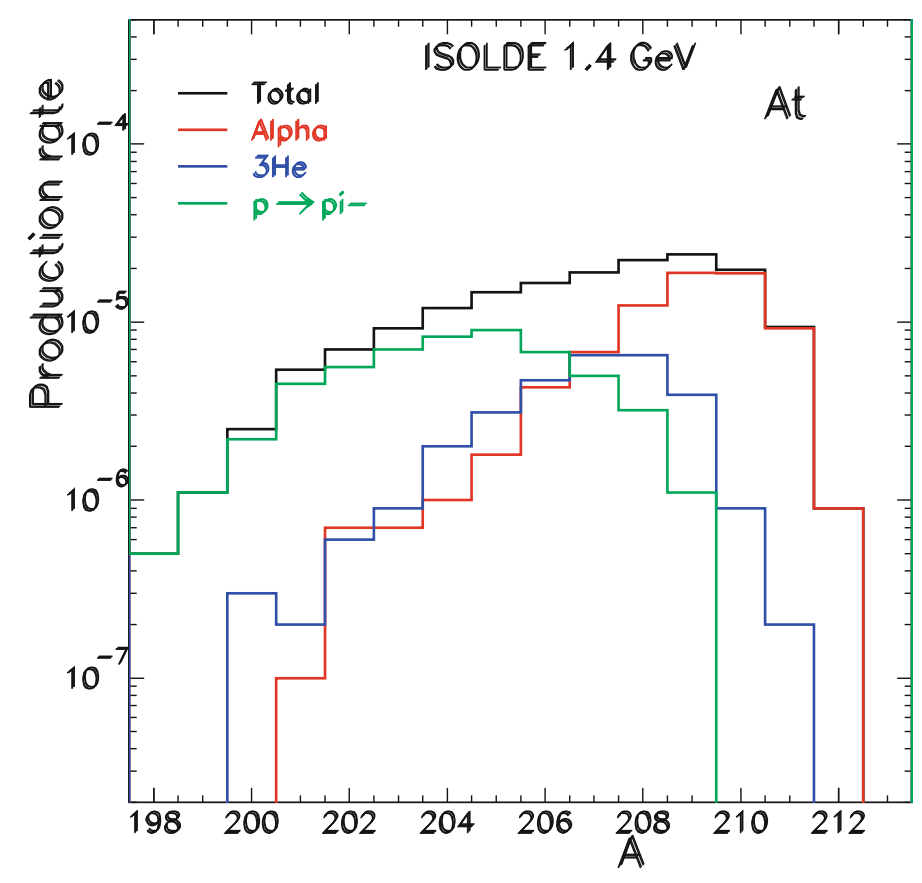

Fig. 15. Number of nuclei produced per incident proton for the different astatine isotopes as predicted by INCL4.6-ABLA07 implemented in MCNPX for the ISOLDE LBE target irradiated by $1.4 \mathrm{GeV}$ protons. Black: total production; green: production through $\left(p, \pi^{-} x n\right)$ reactions; red and blue: through secondary reactions induced by alpha's and ${ }^{3}$ He, respectively. Adapted from ref. [60].

have been identified. The list includes three $A+1$ isobars, three $A$ isobars and two $A-1$ isobars, $A$ being the mass number of the target nucleus. For each of these nucleides, the reaction mechanism has been identified. Depending on the mass number the experimental production cross sections may vary by orders of magnitude. It should be stressed that the predictions of INCL4 for the production of these residues give correctly the order of magnitude. It nevertheless remains that for many of these cross sections and for other observables indicated in this paper, the INCL4 fails by factors of the order of 3,4 or so, while for the rest of the observables, the predictions are (globally) better. To be short, the INCL4 model is satisfactory for several collision events and not for single collision events.

A tentative explanation is proposed in this paper. It is argued that during collisions, nucleons are basically off the energy shell, whereas INC models are handling on the energy shell nucleons. The off-shell quantum effects can directly influence the evaluation of observables in one collision events. They may not influence the observables linked to several collision events, following the conventional wisdom that quantum effects may be at random and cancel for several collisions and not for a single collision. But there is no proof of this conjecture. This warrants further investigations.

Before closing this section, it is perhaps useful to say a few words about the implications of our considerations. One may wonder whether our conclusions are typical of the INCL model, on which we relied here, or are more general. First of all, one has to acknowledge that the incapibility to cope satisfactorily with single-scattering events is not typical of the INCL model. Many INC models show the same defect, as can be seen from the IAEA Intercomparison [9, 12]. This is not really surprising since the description of a single-scattering event is very similar in many models, as they are using basically the same cross sections and similar angular distributions. Of course, the amplitude of the phenomenon may differ in various INC models. Indeed, although we did not comment on this point, it is easy to realize that the single-scattering events under interest are largely peripheral events. Their frequency may thus be influenced by features like nuclear surface structure, Pauli blocking and/or rescattering properties, which may differ from INC model to INC model, as can be seen from ref. [9]. One may thus safely say that basically all INC models are suffering from the same defect with qualitatively the same amplitude.

As we said in sect. 5, after the discussion following eq. (28), the introduction of quasi-particle effects in INC-like models requires a huge task, probably an order of magnitude larger that the one that led to the development of INC models themselves. It is certainly outside the scope of this paper, even in an elementary way.

But, one has also to keep in mind that there are other possible explanations. For instance, in INC models, binary collisions are considered as point-like avatars, both in space and in time. Since the conditions for independence of successive collisions (see eq. (3)) are marginally fulfilled, a small departure from this point-like avatar hypothesis may have non-negligible consequences. In addition, we have briefly discussed, in sect. 5, the use of another way of parametrizing nuclear densities, which in INCL models, allows for the simulation of other quantum effects. 


\section{References}

1. W. Gudowski, Nucl. Phys. A 654, 436c (1999).

2. H. Aït Abderrahim, P. Baeten, D. De Bruyn, J. Heyse, P. Schuurmans, J. Wagemans, Nucl. Phys. News 20, 24 (2010).

3. D. Filges, F. Goldenbaum, Handbook of Spallation Research Theory, Experiments and Applications (Wiley VCH, Berlin, 2010)

4. Geant4 Collaboration, Nucl. Instrum. Methods Phys. Res. A 506, 250 (2003).

5. I. Tanihata, On the possible use of secondary radioactive beams, in Treatise on Heavy-Ion Science, edited by D.A. Bromley, Vol. 8 (Plenum Press, New York, 1989) p. 443.

6. M. Durante, Riv. Nuovo Cimento 25, 1 (2002).

7. M. Longair, High Energy Astrophysics, Vol. 1 and 2 (Cambridge University Press, Cambridge 1997).

8. G. Kraft, Strahlenther. Onkol. 166, 10 (1990).

9. Benchmark of Spallation Models, organized by the IAEA, http://www-nds.iaea.org/spallations/.

10. J. Cugnon, Few-Body Syst. 53, 181 (2012).

11. D. Filges, S. Leray, Y. Yariv, A. Mengoni, A. Stanculescu, G. Mank, Joint ICTP-IAEA Advanced Workshop on Model Codes for Spallation Reactions, International Centre for Theoretical Physics, Trieste, Italy 4 - 8 February 2008 (IAEA, Vienna, Austria, 2008) (NDS)-0530.

12. J.-C. David, D. Filges, F. Gallmeier, M. Khankader, A. Konobeyev, S. Leray, G. Mank, A. Mengoni, R. Michel, N. Otuka, Y. Yariv, Prog. Nucl. Sci. Technol., Conf. Ser. 2, 942 (2011).

13. J.-P. Meulders, A. Koning, S. Leray, HINDAS Detailed Fianal Report, available on-line at the following address: http://www.theo.phys.ulg.ac.be/ cugnon/Final_Scientific_Report_HINDAS.pdf.

14. E. M. Gonzalez, A. Koning, S. Leray, A. Plompen, J. Sanz, NUDATRA, Nuclear Data for Transmutation in IP-Eurotrans, available on-line at the following address: ftp://ftp.nrg.eu/pub/www/talys/bib_Koning/2006_Gonzalez_NUDATRA_Nimes.pdf.

15. European Union research contract ANDES-N² 249671 (RTD-J5/AC/MC:ap/Ares) (2010) 662150.

16. J. Cugnon, C. Volant, S. Vuillier, Nucl. Phys. A 620, 475 (1997).

17. R. Serber, Phys. Rev. 72, 1114 (1947).

18. S. Vuillier, $\mathrm{PhD}$ thesis, Paris-Sud University, France, 1998

19. A. Boudard, J. Cugnon, S. Leray, C. Volant, Phys. Rev. C 66, 044615 (2002).

20. A. Boudard, J. Cugnon, in Proceedings of the Joint ICTP-IAEA Advanced Workshop on Model Codes for Spallation Reactions, ICTP, Trieste, 4-8 February 2008, edited by D. Filges et al., IAEA INDC (NDS)-0530 (IAEA Publications, Vienna, Autriche 2008) pp. 29-50.

21. S.G. Mashnik, K.K. Gudima, R.E. Prael et al., in Proceedings of the Joint ICTP-IAEA Advanced Workshop on Model Codes for Spallation Reactions, ICTP, Trieste, 4-8 February 2008, edited by D. Filges et al., IAEA INDC (NDS)-0530 (IAEA Publications, Vienna, Autriche 2008) pp. 51-52.

22. Y. Yariv, Th. Aoust, A. Boudard, J. Cugnon, J.-C. David, S. Lemaire, S. Leray, in Proceedings of the International Conference on Nuclear Data for Science and Technology, edited O. Bersillon et al. (EDP Sciences, Paris, 2008) pp. 1125-1128.

23. J. Cugnon, A. Boudard, S. Leray, D. Mancusi, in Proceedings of Int. Topical Meeting on Nuclear Research Applications and Utilization of Accelerators (AccApp09), IAEA, Vienna, 2009 (IAEA Publications, Vienna, 2010) ISBN 978-92-0-150410-4, SM/SR-02.

24. A. Boudard, J. Cugnon, J.-C. David, S. Leray, D. Mancusi, Phys. Rev. C 87, 014606 (2013).

25. L. Audirac et al., Phys. Rev. C 88, 041602 (2013).

26. D. Mancusi, A. Boudard, J. Carbonell, J. Cugnon, J.-C. David, S. Leray, Phys. Rev. C 91, 034602 (2015).

27. A. Kelić, M.V. Ricciardi, K.-H. Schmidt, in Proceedings of the Joint ICTP-IAEA Advanced Workshop on Model Codes for Spallation Reactions, in IAEA INDC (NDS)-0530, edited by D. Filges et al. (IAEA Publications, Vienna, 2008) pp. 181-222.

28. D. Mancusi, A. Boudard, J. Cugnon, J.-C. David, P. Kaitaniemi, S. Leray, Phys. Rev. C 91, 054602 (2014).

29. L.P. Kadanoff, G. Baym, Quantum Statistical Mechanics (W. A. Benjamin, New York, 1962).

30. W. Botermans, R. Malfliet, Phys. Lett. B 171, 22 (1986).

31. W. Botermans, R. Malfliet, Phys. Rep. 198, 115 (1990).

32. V.E. Bunakov, G.V. Matvejev, Z. Phys. A 322, 511 (1985).

33. A.S. Iljinov, M.V. Kazarnovsky, E.Ya. Paryev, Intermediate-Energy Nuclear Physics (CRC Press Inc, London, 1994).

34. R.E. Chrien et al., Phys. Rev. C 21, 1014 (1980).

35. J.A. McGill, G.W. Hoffmann, M.L. Barlett, R.W. Fergerson, E.C. Milner, R.E. Chrien, R.J. Sutter, T. Kozlowski, R.L. Stearns, Phys. Rev. C 29, 204 (1984).

36. X. Ledoux et al., Phys. Rev. Lett. 82, 4412 (1999).

37. J. Cugnon, S. Leray, E. Martinez, Y. Patin, S. Vuillier, Phys. Rev. C 56, 2431 (1997).

38. A.K. Weaver, J.D. Anderson, H.H. Barschall, J.C. Davis, Phys. Med. Biol. 18, 64 (1973).

39. S. Hashimoto, Y. Iwamoto, T. Sato, K. Niita, A. Boudard, J. Cugnon, J.-C. David, S. Leray, D. Mancusi, Nucl. Instrum. Methods Phys. Res. B 333, 27 (2014).

40. E.L. Hjort et al., Phys. Rev. C 53, 237 (1996).

41. M. Hagiwara, T. Itoga, N. Kawata, N. Hirabayashi, T. Oishi, T. Yamauchi, M. Baba, M. Sugimoto, T. Muroga, Fusion Sci. Technol. 48, 1320 (2005).

42. S. Chiba et al., Phys. Rev. C 54, 285 (1996). 
43. T. Enqvist et al., Nucl. Phys. A 686, 481 (2001).

44. A. Kelić et al., Phys. Rev. C 70, 064608 (2004).

45. N.P. Jacob, S.S. Markowitz, Phys. Rev. C 11, 541 (1975).

46. C.-X. Chen et al., Phys. Rev. C 56, 1536 (1997).

47. C. Villagrasa-Canton et al., Phys. Rev. C 75, 044603 (2007).

48. F. Rejmund et al., Nucl. Phys. A 683, 540 (2001).

49. L. Giot et al., Nucl. Phys. A 899, 116 (2013).

50. P. Napolitani et al., Phys. Rev. C 76, 064609 (2007).

51. L. Audouin et al., Nucl. Phys. A 768, 1 (2006).

52. J. Taïeb et al., Nucl. Phys. A 724, 413 (2003).

53. Y.E. Titarenko et al., Experimental and Theoretical Study of the Yields of Residual Product Nuclei Produced in Thin Targets Irradiated by 100-2600 MeV Protons, INDC report INDC(CCP)-434 (IAEA, Nuclear Data Section, International Nuclear Data Committee, Vienna, Austria, 2002).

54. R. Michel et al., Nucl. Instrum. Methods B 103, 183 (1995).

55. P.L. Reeder, Phys. Rev. 178, 1795 (1969).

56. Th. Aoust, J. Cugnon, Nucl. Phys. A 828, 52 (2009).

57. T.E. Ward, P.P. Singh, D.L. Friesel, A. Yavin, A. Doron, J.M. D’Auria, G. Sheffer, M. Dillig, Phys. Rev. C 24, 588 (1981).

58. Th. Aoust, J. Cugnon, Phys. Rev. C 74, 064607 (2006).

59. M. Dombsky et al., Phys. Rev. C 32, 253 (1985).

60. J.-C. David, A. Boudard, J. Cugnon, S. Ghali, S. Leray, D. Mancusi, L. Zanini, Eur. Phys. J. A 49, 29 (2013).

61. J. Cugnon, P. Henrotte, Nuclear Reactions Mechanisms: from Compound Nucleus to Multiple Scattering, in Lecture Notes (Université de Louvain-la-Neuve, Belgium, July, 2002).

62. R.J. Glauber, in Lectures in Theoretical Physics, Vol. I, edited by W. Brittin (Interscience, New York, 1959).

63. K.M. Watson, Phys. Rev. 89, 575 (1953).

64. M. Goldberger, K.M. Watson, Collision Theory (Wiley, New York, 1964)

65. A.K. Kerman, H. McManus, R.M. Thaler, Ann. Phys. (N.Y.) 8, 551 (1959).

66. H. Feshbach, J. Hüfner, Ann. Phys. (N.Y.) 56, 268 (1970).

67. J. Cugnon, B. Vandenbossche, Internal report, University of Liège, 2015, available on-line at the following address: http://www. theo.phys.ulg.ac.be/wiki/uploads/d/d9/SSA.pdf.

68. E.F. Redish, K. Stricker-Bauer, Phys. Rev. C 36, 513 (1987).

69. S. Leray, private communication.

70. J. Cugnon, B. Vandenbossche, Internal report, University of Liège, 2015, available on-line at the following address: http://www . theo.phys.ulg.ac.be/wiki/uploads/6/66/SpallQE.pdf.

71. S.J. Wallace, Phys. Rev. C 12, 179 (1975).

72. M.A. Nagarajan, W.L. Wang, D.J. Ernst, R.M. Thaler, Phys. Rev. C 11, 1167 (1975).

73. J. Cugnon, A. Lejeune, P Grangé, Phys. Rev. C 35, 861 (1987).

74. C.Q. Li, R. Machleidt, Phys. Rev. C 48, 1702 (1993).

75. C.Q. Li, R. Machleidt, Phys. Rev. C 49, 566 (1994).

76. J. Cugnon, R. Sartor, unpublished.

77. B. ter Haar, R. Malfliet, Phys. Rev. C 36, 1611 (1987).

78. H. Takada, J. Nucl. Sci. Tech. 33, 275 (1996).

79. W.M. Alberico, M. Ericson, A. Molinari, Nucl. Phys. A 379, 429 (1982).

80. M. Ichimura, K. Kawahigashi, T.S. Jørgensen, C. Gaarde, Phys. Rev. C 39, 1446 (1989).

81. H. Esbensen, G.F. Bertsch, Ann. Phys. (N.Y.) 157, 255 (1984).

82. H. Esbensen, G.F. Bertsch, Phys. Rev. Lett. 52, 2257 (1984).

83. D.L. Prout et al., Phys. Rev. C 52, 228 (1995).

84. P.K.A. de Witt Huberts, J. Phys. G: Nucl. Part. Phys. 16, 507 (1990).

85. P. Danielewicz, Ann. Phys. (N.Y.) 152, 239 (1984).

86. P. Danielewicz, Ann. Phys. (N.Y.) 152, 305 (1984).

87. R. Malfliet, Nucl. Phys. A 545, 3c (1992).

88. ANDES final report, http://cordis .europa.eu/result/rcn/149587_en.html.

89. L. Zanini et al., in International Conference on Nuclear Data for Science and Technology, edited by R.C. Haight et al. (Melville, New York, 2005) p. 1525.

90. Y. Tall et al., in Proceedings of the International Conference on Nuclear Data for Science and Technology, April 22-27, 2007, Nice, France, edited by O. Bersillon, F. Gunsing, E. Bauge, R. Jacqmin, S. Leray (EDP Sciences, Paris, 2008) p. 1069.

91. Y. Tall, PhD Thesis, Université de Nantes, France (2008).

92. L. Zanini et al., in preparation.

93. Th. Aoust, PhD thesis, University of Liège (2007).

94. Th. Aoust, J. Cugnon, J. Wagemans, in Proceedings of the International Conference on Nuclear Data for Science and Technology, edited by O. Bersillon et al. (EDP Sciences, Paris, 2008) pp. 1201-1204.

95. S.G. Mashnik et al., J. Phys.: Conf. Ser. 41, 340 (2006).

96. N. Metropolis et al., Phys. Rev. 110, 185 (1958).

97. N. Metropolis et al., Phys. Rev. 110, 204 (1958).

98. I. Tanihata, S. Nagamiya, S. Schnetzer, H. Steiner, Phys. Lett. B 100, 121 (1981). 